4

\title{
Numerical and Experimental Analysis of Shallow Turbulent Flow over
} Complex Roughness Beds

Yong Zhang, State Key Laboratory of Hydroscience and Engineering, Tsinghua University, Beijing 100084, China

\section{Email: zhang.yong.sichuan@163.com}

Matteo Rubinato, Faculty of Engineering, Environment \& Computing, School of Energy, Construction and Environment, Coventry University, Coventry, CV1 5FB, UK

Email: matteo.rubinato@.coventry.ac.uk ORCiDorcid.org/0000-0002-8446-4448

Ehsan Kazemi, Department of Civil and Structural Engineering, The University of Sheffield, Sheffield S1 3JD, UK

Email: e.kazemi@,sheffield.ac.uk, ORCiD: orcid.org/0000-0002-1780-1846

Jaan $\mathrm{H} . \mathrm{Pu}$, Faculty of Engineering and Informatics, University of Bradford, Bradford, BD7 $1 D P, U K$

Email: J.H.Pu1@bradford.ac.uk ORCiD: orcid.org/0000-0002-3944-8801

Yuefei Huang, State Key Laboratory of Hydroscience and Engineering, Tsinghua University, Beijing 100084, China

Email:yuefeihuang@tsinghua.edu.cn

Pengzhi Lin, State Key Laboratory of Hydraulics and Mountain River Engineering, Sichuan University, Chengdu 610065, China

Email: cvelinpz@scu.edu.cn (corresponding author)

\section{ABSTRACT}

A set of shallow-water equations (SWEs) based on a $\hat{k}-\hat{\varepsilon}$ Reynold stress model is established to simulate the turbulent flows over a complex roughness bed. The fundamental equations are discretized by the second-order finite-difference method (FDM), in which spatial and temporal discretization are conducted by staggered-grid and leap-frog schemes, respectively. The turbulent model in this study stems from the standard $\hat{k}-\hat{\varepsilon}$ model, but is enhanced by replacing the conventional vertical production with a more rigorous and precise generation derived from the energy spectrum and turbulence scales. To verify its effectiveness, the model is applied to 
compute the turbulence in complex flow surroundings (including a rough bed) in an abrupt bend and in a natural waterway. The comparison of the model results against experimental data and other numerical results shows the robustness and accuracy of the present model in describing hydrodynamic characteristics, especially turbulence features on the complex roughness bottom.

Keywords: Energy spectrum, Roughness bed, SWE model, Shallow flows, Turbulent flows

\section{Introduction}

Large and small turbulent swirling flows are often clearly observable when dealing with hydraulic structures in rivers and coastal areas and they are a key factor that influence the frequency and magnitude of natural processes such as the sediment transport, mixing of pollutants and/or riverbed deformation. Therefore, to better manage the rivers and design reliable hydraulic structures, it is fundamental to understand these features and facilitate their predictions. However, certain external factors (e.g. various and inconsistent boundary conditions) make the characterizations of turbulent flows very challenging. To the author's knowledge, there is not yet a fully accurate, time-convenient, or general numerical model to completely replicate the turbulent flows and their impacts over a natural roughness bed. Despite that, effective simulations of the turbulent flow for some specific scenarios have been made due to the rapid progress on the numerical modeling techniques and the computing powers.

The nature of turbulence is fundamentally three-dimensional (3D). Historically, 3D approaches on the turbulence modeling mainly included the Direct Numerical Simulation (DNS), LargeEddy Simulation (LES), and Reynolds-Averaged Navier-Stokes (RANS) modeling [1-4]. Along the rivers and coastal regions, the flow domain is quite complex and spacious, and hence to characterize the flow structures it would be excessively time-consuming to apply any of these three approaches, which would require a large number of grid nodes in order to provide the accurate results [5]. The two-dimensional (2D) Shallow Water Equations (SWE) coupled with the benchmark turbulence closure model is much faster and enables the interpretation of turbulent characteristics using a smaller vertical length scale $(z)$ as compared with the two horizontal ones ( $x$ and $y$ ) in those regions [6-9]. Furthermore, to obtain more accurate and repeatable results, it is also critical to select the appropriate coefficients in these turbulence closure equations. 
2D shallow-turbulence flow models have been extensively developed over the last decades. Most of the available ones are based on the Boussinesq approximations. For example, the depthaveraged eddy viscosity model suggests that eddy viscosity is the simple product of the bed shear velocity and water depth [10] and the depth-averaged mixing length model accounts for the influence of vertical turbulence [11]. Rastogi and Rodi [12] established the 2D standard depth-averaged $\hat{k}-\hat{\varepsilon}$ turbulence model based on the 3D version described by Launder and Spalding [13]. To widen the range of practical applications, some coefficients in the model developed by Rastogi and Rodi were modified and new $\hat{k}-\hat{\varepsilon}$ models were introduced, as presented in the other studies [14-18]. The standard $\hat{k}-\hat{\varepsilon}$ model has been demonstrated to provide satisfactory results after numerous comparisons with the measured data $[11,14]$ and it is also relatively simple to use and very fast. Despite this, more progresses have been made in this field and recently, Cea et al. [11] established a depth-averaged algebraic stress turbulence model (DASM) to solve a single transport equation for each Reynolds stress without requiring an isotropic assumption.

For the shallow turbulent flows on a roughness bed, the vertical velocity gradient distribution is the main source of turbulence. The accuracy of its depth-averaged process directly determines the numerical performance of the aforementioned $\hat{k}-\hat{\varepsilon}$ models. To date there is still area for improvement due to the fact that preliminary results of the standard $\hat{k}-\hat{\varepsilon}$ model only partially agree with the experimental data [12]. According to Rastogi and Rodi [12], this may be related to the bottom shear stress, via the friction velocity, under the assumption of similarity in the vertical velocity profiles. The depth-averaging process is a consideration only of the macroscopic effects of the bottom roughness on the turbulent generations. In the classic "cascade" theory of energy introduced by Richardson [19], the turbulent motion is a process of energy transfer among various scales including not only the macroscale, but also various microscales $[2,20,21]$. The contribution of these features should be rigorously and precisely replicated within turbulent closure models.

The turbulent structure of various scales based on the framework of energy cascade can be divided into the energy-containing, inertial, and dissipative regions, respectively. The kinetic energy, produced in the energy-containing region, is considered to be transferred by inertial forces to smaller scales until the energy is typically dissipated by the molecular viscosity [2]. The energy spectrum in the inertial region has a universal statistical form, i.e. the Kolmogorov $-5 / 3$ spectrum [22], and can be applied to larger or smaller wave numbers as the Reynolds number increases [8]. Nezu and Nakagawa [20] analogized the energy transfer processes to 
open-channel flows and divided the whole water depth into the three regions: wall, intermediate, and free-surface zones. A universal log-law in the intermediate region was verified by the extensive experimental and numerical results and they confirmed this can be applied to a wider range of bed roughness and Reynolds numbers.

Taking into considerations all of these previous works and the new insights that have been provided, this study aims to improve the performance of the standard $\hat{k}-\hat{\varepsilon}$ model to characterise the generation of turbulent conditions at various scales over complex roughness beds. The numerical SWE model utilised in this work includes a second-order leap-frog finitedifference method (FDM) and it is built into a staggered-grid system. This model was initially developed by Cho [23] to calculate the evolution of the long waves and was further extended by Lin [24] to simulate the turbulent structures within an experimental zone. Lately this turbulence model has been widely used to compute the complex flows induced by irregular geometries and the results obtained have proven that it is a robust numerical technique [25-27]. In the present study, we emphasized the improvement of the model for application to the complex roughness beds.

The paper is organized as follows: Section 2 presents the description of the mathematical and numerical shallow turbulence model considered for this study, clarifying various assumptions and hypothesis: the governing equations are presented in Section 2.1, then the vertical turbulent production is formulated in Section 2.2 by incorporating the energy transfer information between various scales into $P_{k V}$ and $P_{\varepsilon V}$ based on the two universal semi-theoretical formulas of Kolmogorov -5/3 scaling law and log-law. Section 3 includes the validation of the numerical model against the experimental data collected on a flume, where varying roughness on the bed was tested with a complex sharp bend. Finally, to further demonstrate the potentials of the model, its performance was more vigorously examined in the transport of moraine along the Yangtze River under dry seasons, and the results are explained in Section 4. Section 5 provides a brief summary and concluding remarks of the whole study.

\section{Numerical Model}

\subsection{Governing equations}

This section presents the governing equations and the boundary conditions utilised in this study.

\subsubsection{Shallow-water equations}

The time-dependent SWEs are the fundamental hydrodynamic equations described in this 
136 section. By integrating the Reynolds Averaged Navier-Stokes (RANS) equations along the 137 entire water depth and assuming the vertical flow to be nearly-uniform and pressure

138 distributions to be hydrostatic, the SWEs can be given as follows [25-27]:

$139 \frac{\partial H}{\partial t}+\frac{\partial P}{\partial x}+\frac{\partial Q}{\partial y}=0$

$$
\frac{\partial P}{\partial t}+\frac{\partial}{\partial x}\left(\frac{P^{2}}{H}\right)+\frac{\partial}{\partial y}\left(\frac{P Q}{H}\right)+g H \frac{\partial \eta}{\partial x}=\frac{1}{\rho} \frac{\partial\left(H T_{x x}\right)}{\partial x}+\frac{1}{\rho} \frac{\partial\left(H T_{y x}\right)}{\partial y}-\frac{f}{8 H^{2}} \sqrt{P^{2}+Q^{2}} P-\frac{2}{3} \frac{\partial(H \hat{k})}{\partial x}(1 \mathrm{~b})
$$

$$
\frac{\partial Q}{\partial t}+\frac{\partial}{\partial x}\left(\frac{P Q}{H}\right)+\frac{\partial}{\partial y}\left(\frac{Q^{2}}{H}\right)+g H \frac{\partial \eta}{\partial y}=\frac{1}{\rho} \frac{\partial\left(H T_{x y}\right)}{\partial x}+\frac{1}{\rho} \frac{\partial\left(H T_{y y}\right)}{\partial y}-\frac{f}{8 H^{2}} \sqrt{P^{2}+Q^{2}} Q-\frac{2}{3} \frac{\partial(H \hat{k})}{\partial y}(1 \mathrm{c})
$$

142 where $H\left(=\eta-z_{b}\right)$ is the water depth, in which $\eta$ and $z_{b}$ are the free surface and bed elevation, 143 respectively (Fig. 1); $P(=H U)$ and $Q(=H V)$ are components of unit volume flux, in which $144 U\left(=\frac{1}{H} \int_{z_{b}}^{\eta}\langle u\rangle d z\right)$ and $V\left(=\frac{1}{H} \int_{z_{b}}^{\eta}\langle v\rangle d z\right)$ are the depth-averaged velocities in $x$ and $y$ directions,

145 respectively; $g$ is gravitational acceleration; $\rho$ is fluid density; $\hat{k}\left(=\frac{1}{H} \int_{z_{b}}^{\eta} k d z\right)$ is the depth146 averaged turbulent energy; $T_{x x}, T_{y y}, T_{y x}$, and $T_{x y}$ are the depth-averaged effective stresses 147 expressed as follows [25-26]:

$148 T_{x x}=2 \rho \frac{v+\hat{v}_{t}}{H} \frac{\partial P}{\partial x}$

$$
T_{y y}=2 \rho \frac{v+\hat{v}_{t}}{H} \frac{\partial Q}{\partial y}
$$

$$
T_{y x}=T_{x y}=\rho \frac{v+\hat{v}_{t}}{H}\left(\frac{\partial P}{\partial y}+\frac{\partial Q}{\partial x}\right)
$$

151 where $v$ and $\hat{v}_{t}$ are the kinematic viscosity and the depth-averaged turbulent viscosity, 152 respectively, as defined in Eq. (4).

$154 f$ in Eq. (1) is the bed friction factor defined according to parameters for the smooth and rough 155 beds previously obtained [26, 28], such as Chézy-Manning's and Altsul's formula:

$156 f=\frac{8 g m^{2}}{H^{1 / 3}}$ 
b)

159 where $m$ is Manning's roughness coefficient; $k_{s}$ is bed roughness height, which in this study

160 it is considered to be the median diameter of the bed material; $R$ is the hydraulic radius (the water depth, in this study); and $R e$ is the Reynolds number.

\subsubsection{Depth-averaged $\hat{k}-\hat{\varepsilon}$ closure equations}

163 The depth-averaged velocity field in Eq. (1) can be solved when $\hat{v}_{t}$ is determined. The

164 specification of $\hat{v}_{t}$ is assumed to be mathematically analogous to the turbulent viscosity $v_{t}$ in 165 the standard $k-\varepsilon$ model [25], and can be expressed as follows:

$166 \hat{v}_{t}=C_{\mu} \frac{\hat{k}^{2}}{\hat{\varepsilon}}$

167 The transport equations $\hat{k}$ and $\hat{\varepsilon}$ can be then introduced, respectively, as follows:

168

$$
\begin{gathered}
\frac{\partial(H \hat{k})}{\partial t}+\frac{\partial(P \hat{k})}{\partial x}+\frac{\partial(Q \hat{k})}{\partial y}=H \hat{v}_{t}\left[2\left(\frac{\partial U}{\partial x}\right)^{2}+2\left(\frac{\partial V}{\partial y}\right)^{2}+\left(\frac{\partial U}{\partial y}+\frac{\partial V}{\partial x}\right)^{2}\right]+P_{k V}+ \\
\frac{\partial}{\partial x}\left[\left(v+\frac{\hat{v}_{t}}{\sigma_{k}}\right) \frac{\partial(H \hat{k})}{\partial x}\right]+\frac{\partial}{\partial y}\left[\left(v+\frac{\hat{v}_{t}}{\sigma_{k}}\right) \frac{\partial(H \hat{k})}{\partial y}\right]-H \hat{\varepsilon}
\end{gathered}
$$

169

$\frac{\partial(H \hat{\varepsilon})}{\partial t}+\frac{\partial(P \hat{\varepsilon})}{\partial x}+\frac{\partial(Q \hat{\varepsilon})}{\partial y}=C_{\varepsilon 1} \frac{\hat{\varepsilon}}{\hat{k}} H \hat{v}_{t}\left[2\left(\frac{\partial U}{\partial x}\right)^{2}+2\left(\frac{\partial V}{\partial y}\right)^{2}+\left(\frac{\partial U}{\partial y}+\frac{\partial V}{\partial x}\right)^{2}\right]+$

$$
P_{\varepsilon V}+\frac{\partial}{\partial x}\left[\left(v+\frac{\hat{v}_{t}}{\sigma_{\varepsilon}}\right) \frac{\partial(H \hat{\varepsilon})}{\partial x}\right]+\frac{\partial}{\partial y}\left[\left(v+\frac{\hat{v}_{t}}{\sigma_{\varepsilon}}\right) \frac{\partial(H \hat{\varepsilon})}{\partial y}\right]-C_{\varepsilon 2} H \frac{\hat{\varepsilon}^{2}}{\hat{k}}
$$

170 where $C_{\mu}, \sigma_{k}, \sigma_{\varepsilon}, C_{1 \varepsilon}$ and $C_{2 \varepsilon}$ are the empirical constants and their values, as recommended by

171 Launder and Spalding [13], are

$$
C_{\mu}=0.09, \sigma_{k}=1.0, \sigma_{\varepsilon}=1.3, C_{1 \varepsilon}=1.44, C_{2 \varepsilon}=1.92
$$

173 and, as suggested in [8], [12] and [13],

$174 \quad P_{k V}=\frac{n^{2} g}{H^{\frac{1}{3}}}\left(U^{2}+V^{2}\right)^{\frac{3}{2}}$

$175 \quad P_{\varepsilon V}=\frac{C_{2} C_{\mu} n^{\frac{5}{2}} g^{\frac{5}{4}}\left(U^{2}+V^{2}\right)^{2}}{H^{\frac{17}{12}}}$ 


\subsection{Vertical turbulent production $P_{k V}$ and $P_{\varepsilon V}$}

$178 P_{k V}$ and $P_{\varepsilon V}$ in Eq. (5) are the components generated by turbulence and can be defined as the 179 tensor product of the horizontal Reynolds stress and vertical velocity gradient, respectively. In 180 the context of homogeneous, open-channel turbulence, they represent all the turbulent energy 181 produced. The corresponding expressions to calculate their magnitude were deduced by Rastogi 182 and Rodi [12], although their derivations seemed to have overgeneralized the generation of 183 vertical turbulent features.

According to the theory of cascade energy, which has been proven and validated against extensive experimental studies, the turbulent motion is a one-way energy transfer process from large scale eddies to smaller ones [2]. The theory can also be applied to the transfer of energy from the bottom of the river to the free surface [20]. To rigorously and precisely quantify the energy transfer across various scales, this study presents an additional model that estimates the generation of vertical turbulent features in a different manner, considering the interaction between points at different levels and the total energy spectrum. Bottom roughness elements markedly affect the amount of turbulence. Turbulence tends to be isotropy as the bed roughness and Reynolds numbers increase [2, 20,29]. Cea et al. [11] found a similar degree of accuracy between the $\hat{k}-\hat{\varepsilon}$ model, which is based on the concise isotropic hypothesis, and the DASM model, which includes complex model structures considering anisotropy. Based on this assumption, below are listed the steps selected to deduce the expressions of $P_{k V}$ and $P_{\varepsilon V}$.

\subsubsection{Mean velocity profiles}

For a uniform and fully-developed turbulent flow in a wide open channel, the Reynolds equation in the $z$-direction (as derived from the Navier-Stokes equations) is reduced to:

$\rho v \frac{d\langle u\rangle}{d z}-\rho\left\langle u^{\prime} w^{\prime}\right\rangle=\rho u_{*}^{2}\left(1-\frac{z}{H}\right)$

201 where $\rho v d\langle u\rangle / d z$ is the viscous stress; $-\rho\left\langle u^{\prime} w^{\prime}\right\rangle$ is the Reynolds stress; $u_{*}\left(=\sqrt{\tau_{b} / \rho}\right)$ is the friction velocity, in which $\tau_{b}$ is the bed shear stress at $z=0$.

By considering the Prandtl's mixing-length hypothesis, $v$ can be expressed as the product of a velocity scale $u^{*}$ and a length scale $l_{m}$, in which $u^{*}$ is specified as $u^{*}=\left|\left\langle u^{\prime} w^{\prime}\right\rangle\right|^{1 / 2}$. From Eq. (8), 
the following equation could be obtained:

$$
\frac{d\langle u\rangle^{+}}{d z^{+}}=\frac{2 \tau / \tau_{b}}{1+\sqrt{1+\left(4 \tau / \tau_{b}\right)\left(l_{m}^{+}\right)^{2}}}
$$

where $\langle u\rangle^{+}=\langle u\rangle / u_{*}$ and $z^{+}=z u_{*} / v_{k}$ are the dimensionless velocity and z-coordinate normalized by the viscous length $\delta_{v}=v_{k} / u_{*}$, respectively; $l_{m}^{+}=l_{m} u_{*} / v_{k}$ is the dimensionless mixing-length. If appropriate specification of $l_{m}^{+}$over the whole depth can be obtained, Eq. (9) can be easily integrated to yield the distribution of $\langle u\rangle$.

In various regions from $z=0$ to $z=\eta$, the effect of $\rho v d\langle u\rangle / d z$ and $-\rho\left\langle u^{\prime} w^{\prime}\right\rangle$ on the velocity distribution is different. According to their contribution to the turbulent structure, the vertical turbulence fields of $\langle u\rangle$ are divided into two regions: the inner region and the outer region [20]. It is assumed that the viscous effect dominates the inner region (in general, $z / H<(0.15-0.2)$ ), so the length scale can be denoted by $\delta_{v}$. The component $\tau / \tau_{w}$ tends towards unity and $l_{m}^{+}$can be specified as $l_{m}^{+}=\kappa z^{+}$in this region, so the solution to Eq. (9) (the "log-law"), can be obtained as follows:

$\langle u\rangle^{+}=\frac{1}{\kappa} \ln z^{+}+A$

where $\kappa=0.40-0.43$ and $B=5.2$ are the empirical constants $[2,30]$.

It is assumed that the viscous contribution in the outer region (generally, $z / H \geq(0.15-0.2)$ ) to the turbulent structure is negligible, and that $l_{m}^{+}$no longer depends on $\delta_{v}$. It is challenging to solve Eq. (9) because there is no universal representation of $l_{m}^{+}$. The wake law provided by Coles [31], is typically used to extend the log-law to the outer region. The profile of $\langle u\rangle$ in this region can be approximated as follows:

$\langle u\rangle^{+}=\frac{1}{\kappa} \ln z^{+}+A+\frac{2 \Pi}{\kappa} \sin ^{2}\left(\frac{\pi z}{2 H}\right)$

where $\Pi$ is Coles's wake-strength parameter. $\Pi$ has been investigated in various studies where its value has been suggested to be around 0.08-0.20. $\Pi$ also shows no distinct value for flows with different bed roughness conditions [32]. Considering the comparisons made by Pope [2] and Nezu and Nakagawa [20], the profile of $\langle u\rangle$ can be effectively approximated by Eq. (11) over the whole depth except in a small region near the bed. In the core region of closed-bed 
flow (e.g., $z^{+}<26$ ), $l_{m}^{+}$is strongly affected by the Reynolds number and bed roughness, so

$235\langle u\rangle$ is likely to remain the complex profile. In this study, we considered the distribution of $\langle u\rangle$

236 in this region to be determined by using Eq. (11).

\subsubsection{Depth-averaged vertical production}

238 Based on Kolmogorov's scaling theory outlined at [22], the dissipation rate $\varepsilon$ could be related to $\left\|u^{\prime}\right\|$ by using the macroscale of turbulence described in [20,21] as follows:

$240 \varepsilon=K \frac{\left\|u^{\prime}\right\|^{3}}{L_{x}}$

241 where $K=\left[2 /\left(\pi C^{\prime}\right)\right]^{3 / 2}\left(L_{x} k_{0}\right)^{5 / 2}$ is a turbulent coefficient which is greatly influenced by the

242 Reynolds number, $C^{\prime}$ is a universal Kolmogorov constant, $L_{x}$ is the longitudinal integral 243 macroscale, $k_{0}$ is macroscale of turbulence. Nakagawa et al. [33] suggested that $\left\|u^{\prime}\right\| / u_{*}$ can be 244 approximated as the power function of $z / H$ :

$\frac{\left\|u^{\prime}\right\|}{u_{*}}=\left[\frac{B_{N}}{\kappa K}\left(1-\frac{z}{H}\right)\right]^{1 / 3}\left(\frac{z}{H}\right)^{-1 / 6}$

246 Further, under an assumption of isotropy, Nezu and Nakagawa [20] found that the correlation coefficients of turbulence intensities are nearly constant as long as $z^{+}$is sufficiently large. This can be approximately represented by:

where $C_{1}$ and $C_{2}$ are empirical constants.

For stationary and homogeneous turbulence, the turbulent production $P_{k}\left(=-\left\langle u^{\prime} w^{\prime}\right\rangle \partial\langle u\rangle / \partial z\right)$ is balanced approximately by the sum of the turbulent diffusion $D_{k}\left(=(d / d z)\left[\left(v / \sigma_{k}\right)(d k / d z)\right]\right)$ and dissipation $\varepsilon$ independently of the molecular diffusion. In the inertial subrange, energy transfer is the only significant process; there is no energy production or dissipation. Thus,

$256 P_{k} \approx \varepsilon$. The dynamic equilibrium of turbulent energy can then be expressed as follows:

in which $v\left(=C_{\mu} k^{2} / \varepsilon\right)$ is the turbulent viscosity. Substituting Eq. (12) into $v$ (where $L_{x}$ is of the same order as $H$ ) and making use of Eq. (14) (where $u^{\prime}$ is of the order of $u_{*}$ ) yields the 
following approximation of $v$ :

$261 \quad v \approx \frac{1}{k} H u_{*}{ }^{3}$

262 Substituting Eq. (16) into Eq. (15) with the integral from 0 to $z$ yields the distribution of $k$ :

$$
\frac{k}{u_{*}^{2}}=D \exp \left(-2 C_{k} \frac{z}{H}\right)
$$

264 where $D$ and $C_{k}$ are the constants. Applying Eqs. (14) and (17) provides the profiles of $u^{\prime}, v^{\prime}$ 265 , and $w^{\prime}$ :

$$
\frac{\left\|u^{\prime}\right\|}{u_{*}}=D_{u} \exp \left(-C_{k} \frac{z}{H}\right)
$$

$$
\frac{\left\|v^{\prime}\right\|}{u_{*}}=D_{v} \exp \left(-C_{k} \frac{z}{H}\right)
$$

$$
\frac{\left\|w^{\prime}\right\|}{u_{*}}=D_{w} \exp \left(-C_{k} \frac{z}{H}\right)
$$

where $D_{u}, D_{v}, D_{w}$, and $C_{k}$ are the empirical constants; their values are $2.30,1.27,1.63$, and 1.0 , respectively [20].

By closely examining the turbulence intensities presented by Nezu and Nakagawa [20], we found close agreement between Eq. (18) results and the experimental values throughout the whole depth apart from a thin layer near the bed. Surprisingly, any deviation between them gradually decreased as $\operatorname{Re}$ and $k_{s}^{+}\left(=k_{s} u_{*} / v_{k}\right)$ increased. To some extent, Eq. (18) is successful in displaying the vertical distribution of turbulence intensity. Integrating Eq. (18) with the given empirical constants allows the depth-averaged turbulent energy $\hat{k}$ to be obtained: $\hat{k}=2.07 u_{*}^{2}$

279 The constant used in Eq. (19) has been found by combining $D_{u}, D_{v}$, and $D_{w}$ at Eqs. (18a) - (18c), 280 suggested in Nezu and Nakagawa [20]. The formulation of $k$ is described in Eq. (37). According 281 to the theory of cascade energy, at the end of the sequence of processes, the dissipation of turbulent energy is accomplished primarily by molecular viscosity. In other words, the energy of open-channel flows is predominantly dissipated in the free surface region. Observed $\varepsilon$ values in this region are less accurate due to the constraints of free-surface fluctuations. In the comparison made by Nakagawa et al. [33], it is very difficult to obtain a universal formula for $\varepsilon$. In this study, we used $v$ as a replacement for $\varepsilon$ to determine $P_{k V}$ and $P_{\varepsilon V}$. 
$288 \quad v=\frac{\kappa(1-z / H) H u_{*}}{(H / z)+\pi \Pi \sin (\pi z / H)}$

289 Eq. (20) is found from substituting Coles' law at Eq. (11) into Eq. (8). By integrating Eq. (20)

290 from the bed to free surface, we obtained the depth-averaged turbulent viscosity $\hat{v}_{t}$ as follows:

$291 \hat{v}_{t}=0.06 H u_{*}$

292 The constant in Eq. (21) comes from depth-averaging Eq. (20) from bed to free-surface, using

293 described values of $\kappa$ and $\Pi$ in section 2.2.1. In stationary and homogeneous flows, $P_{k V}$ and

$294 P_{\varepsilon V}$ in the $\hat{k}-\hat{\varepsilon}$ transport equations are balanced only by their dissipation terms $\hat{\varepsilon} H$ and

$295 C_{2 \varepsilon} \hat{\varepsilon}^{2} H / \hat{k}$, respectively. $P_{k V}$ and $P_{\varepsilon V}$ can be obtained by rearranging Eq. (5) and combining

296 Eqs. (4), (19) and (21):

$297 P_{k V}=71.42 C_{\mu} u_{*}{ }^{3}$

$298 P_{\varepsilon V}=2464.16 C_{\mu}{ }^{2} C_{2 \varepsilon} \frac{u_{*}{ }^{4}}{H}$

299 where $u_{*}=\sqrt{f\left(U^{2}+V^{2}\right) / 8}$. These Eqs. (22a) and (22b) have been derived from the standard

$300 \hat{k}-\hat{\varepsilon}$ formulations of $P_{k V}$ and $P_{e V}$ terms as showing at Eqs. (7a) and (7b).

301

302

\subsection{Numerical implementation}

303 In this study, we discretized the fundamental equations using the explicit leap-frog FDM which

304 has second-order accuracy in both time and space [25-26]. As plotted in Fig. 2, all of the vectors

$305(P, Q, U$, and $V)$ were evaluated at the faces of the grid; all of the scalars $\left(H, \eta, z_{b}, \hat{k}\right.$, and $\left.\hat{\varepsilon}\right)$

306 were defined at the center of the grid. The defined location of the normal stresses (e.g.,

$307 T_{x x}$ and $T_{y y}$ ) is the same as the scalar; the shear stresses (e.g., $T_{x y}$ and $T_{y x}$ ) were calculated as

308 shown in the right-top corner of the grid.

309

310

311

312

\subsubsection{Continuity equation}

According to the spatial staggered grid system and the temporal leap-frog scheme, the continuity equation is explicitly discretized as follows:

$H_{i, j}^{n+1 / 2}=H_{i, j}^{n-1 / 2}-\frac{\Delta t}{\Delta x_{i}}\left(P_{i+1 / 2, j}^{n}-P_{i-1 / 2, j}^{n}\right)-\frac{\Delta t}{\Delta y_{j}}\left(Q_{i, j+1 / 2}^{n}-Q_{i, j-1 / 2}^{n}\right)$

\subsubsection{Momentum equations}

314 Without loss of generality, only the discretized form of $x$-momentum equation is presented here in detail: 


$$
\begin{aligned}
P_{i+1 / 2, j}^{n+1}= & \frac{1-\frac{f \Delta t}{16\left(H_{i+1 / 2, j}^{n}\right)^{2}}\left[\left(P_{i+1 / 2, j}^{n}\right)^{2}+\left(Q_{i+1 / 2, j}^{n}\right)^{2}\right]^{1 / 2}}{1+\frac{f \Delta t}{16\left(H_{i+1 / 2, j}^{n}\right)^{2}}\left[\left(P_{i+1 / 2, j}^{n}\right)^{2}+\left(Q_{i+1 / 2, j}^{n}\right)^{2}\right]^{1 / 2}} P_{i+1 / 2, j}^{n} \\
& -\frac{\Delta t}{1+\frac{f \Delta t}{16\left(H_{i+1 / 2, j}^{n}\right)^{2}}\left[\left(P_{i+1 / 2, j}^{n}\right)^{2}+\left(Q_{i+1 / 2, j}^{n}\right)^{2}\right]^{1 / 2}}\left\{\left[\frac{\partial}{\partial x}\left(\frac{P^{2}}{H}\right)\right]_{i+1 / 2, j}^{n}+\left[\frac{\partial}{\partial y}\left(\frac{P Q}{H}\right)\right]_{i+1 / 2, j}^{n}\right. \\
& -\frac{g \Delta t}{\left\{1+\frac{f \Delta t}{16\left(H_{i+1 / 2, j}^{n}\right)^{2}}\left[\left(P_{i+1 / 2, j}^{n}\right)^{2}+\left(Q_{i+1 / 2, j}^{n}\right)^{2}\right]^{1 / 2}\right\} \Delta x_{i+1 / 2}} H_{i+1 / 2, j}^{n+1 / 2}\left(\eta_{i+1, j}^{n+1 / 2}-\eta_{i, j}^{n+1 / 2}\right) \\
& +\frac{\Delta t / \rho}{1+\frac{f \Delta t}{16\left(H_{i+1 / 2, j}^{n}\right)^{2}}\left[\left(P_{i+1 / 2, j}^{n}\right)^{2}+\left(Q_{i+1 / 2, j}^{n}\right)^{2}\right]^{1 / 2}}\left[\frac{\partial\left(H T_{x x}\right)}{\partial x}+\frac{\partial\left(H T_{y x}\right)}{\partial y}\right]_{i+1 / 2, j}^{n}
\end{aligned}
$$

317 where the convection terms are respectively discretized as follows by applying the upwind 318 scheme:

319

$$
\begin{aligned}
& {\left[\frac{\partial}{\partial x}\left(\frac{P^{2}}{H}\right)\right]_{i+1 / 2, j}^{n}= \begin{cases}\frac{\left(P_{i+1 / 2, j}^{n}\right)^{2}}{\frac{H_{i+1 / 2, j}^{n}}{\Delta x_{i}}-\frac{\left(P_{i-1 / 2, j}^{n}\right)^{2}}{H_{i-1 / 2, j}^{n}}} & \text { if } \quad P_{i+1 / 2, j}^{n}>0 \\
\frac{\left(P_{i+3 / 2, j}^{n}\right)^{2}}{H_{i+3 / 2, j}^{n}-\frac{\left(P_{i-1 / 2, j}^{n}\right)^{2}}{\Delta x_{i-1 / 2, j}^{n}+\Delta x_{i+1}}} \text { if } \quad P_{i+1 / 2, j}^{n}=0 \\
\frac{\left(P_{i+3 / 2, j}^{n}\right)^{2}-\frac{\left(P_{i+1 / 2, j}^{n}\right)^{2}}{H_{i+1 / 2,2, j}^{n}}}{\Delta x_{i+1}} & \text { if } \quad P_{i+1 / 2, j}^{n}<0\end{cases} }
\end{aligned}
$$

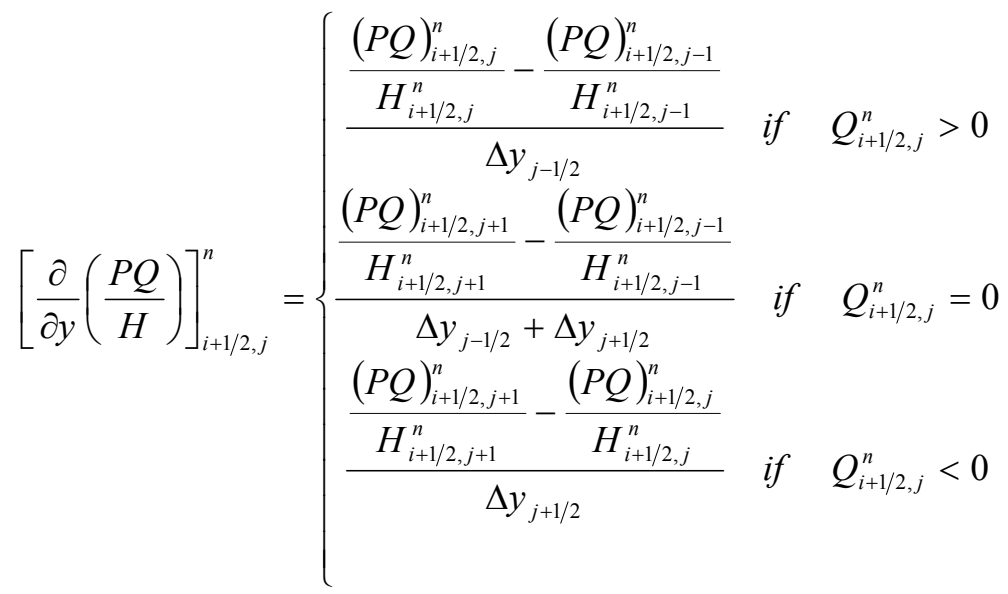

\subsubsection{Turbulent transport equations}

322 The $\hat{\varepsilon}$ - and $\hat{k}$-equations are respectively discretized by applying a semi-implicit scheme: 


$$
\hat{\varepsilon}_{i, j}^{n+1 / 2}=\frac{\frac{(H \hat{\varepsilon})_{i, j}^{n-1 / 2}}{\Delta t}-F \hat{\varepsilon} X-F \hat{\varepsilon} Y+V I S \hat{\varepsilon} X+V I S \hat{\varepsilon} Y+C_{1 \varepsilon} \frac{\hat{\varepsilon}_{i, j}^{n-1 / 2}}{\hat{k}_{i, j}^{n-1 / 2}}\left(P_{h}\right)_{i, j}^{n+1 / 2}+\left(P_{\varepsilon V}\right)_{i, j}^{n+1 / 2}}{\frac{H_{i, j}^{n+1 / 2}}{\Delta t}+C_{2 \varepsilon} \frac{\hat{\varepsilon}_{i, j}^{n-1 / 2}}{\hat{k}_{i, j}^{n-1 / 2}} H_{i, j}^{n-1 / 2}}
$$

$$
\hat{k}_{i, j}^{n+1 / 2}=\frac{\frac{(H \hat{k})_{i, j}^{n-1 / 2}}{\Delta t}-F \hat{k} X-F \hat{k} Y+V I S \hat{k} X+V I S \hat{k} Y+\left(P_{h}\right)_{i, j}^{n+1 / 2}+\left(P_{k V}\right)_{i, j}^{n+1 / 2}}{\frac{H_{i, j}^{n+1 / 2}}{\Delta t}+C_{\mu} \frac{\hat{\varepsilon}_{i, j}^{n-1 / 2}}{\left(\hat{v}_{t}\right)_{i, j}^{n-1 / 2}} H_{i, j}^{n-1 / 2}}
$$

325

Similarly, the upwind scheme can be used to discretize the convective term $F \hat{\varepsilon} X$ (the differences of $F \hat{\varepsilon} Y, F \hat{k} X$, and $F \hat{k} Y$ are similar to $F \hat{\varepsilon} X)$ as follows:

$$
[F \hat{\varepsilon} X]_{i, j}^{n-1 / 2}=\left[\frac{\partial(P \hat{\varepsilon})}{\partial x}\right]_{i, j}^{n-1 / 2}= \begin{cases}\frac{P_{i, j}^{n-1 / 2} \hat{\varepsilon}_{i, j}^{n-1 / 2}-P_{i-1, j}^{n-1 / 2} \hat{\varepsilon}_{i-1, j}^{n-1 / 2}}{\Delta x_{i-1 / 2}} & \text { if } \quad P_{i, j}^{n-1 / 2}>0 \\ \frac{P_{i+1, j}^{n-1 / 2} \hat{\varepsilon}_{i+1, j}^{n-1 / 2}-P_{i-1, j}^{n-1 / 2} \hat{\varepsilon}_{i-1, j}^{n-1 / 2}}{\Delta x_{i-1 / 2}+\Delta x_{i+1 / 2}} & \text { if } \quad P_{i, j}^{n-1 / 2}=0 \\ \frac{P_{i+1, j}^{n-1 / 2} \hat{\varepsilon}_{i+1, j}^{n-1 / 2}-P_{i, j}^{n-1 / 2} \hat{\varepsilon}_{i, j}^{n-1 / 2}}{\Delta x_{i+1 / 2}} & \text { if } \quad P_{i, j}^{n-1 / 2}<0\end{cases}
$$

The central difference method was applied to discretize the diffusion term VIS $\hat{\varepsilon} X$ (the difference forms of VIS $\hat{\varepsilon} Y, V I S \hat{k} X$, and VIS $\hat{k} Y$ are analogous to VIS $\hat{\varepsilon} X$ ) as follows:

$$
\begin{aligned}
& V I S \hat{\varepsilon} X=\frac{1}{\Delta x_{i}}\left\{\left[\left(v_{k}+\frac{\hat{v}_{t}}{\sigma_{\varepsilon}}\right) \frac{\partial(H \hat{\varepsilon})}{\partial x}\right]_{i+1 / 2, j}^{n-1 / 2}-\left[\left(v_{k}+\frac{\hat{v}_{t}}{\sigma_{\varepsilon}}\right) \frac{\partial(H \hat{\varepsilon})}{\partial x}\right]_{i-1 / 2, j}^{n-1 / 2}\right\}= \\
& \frac{1}{\Delta x_{i}}\left[\left(v_{k}+\frac{\left(\hat{v}_{t}\right)_{i+1 / 2, j}^{n-1 / 2}}{\sigma_{\varepsilon}}\right) \frac{H_{i+1, j}^{n-1 / 2} \hat{\varepsilon}_{i+1, j}^{n-1 / 2}-H_{i, j}^{n-1 / 2} \hat{\varepsilon}_{i, j}^{n-1 / 2}}{\Delta x_{i+1 / 2}}-\left(v_{k}+\frac{\left(\hat{v}_{t}\right)_{i-1 / 2, j}^{n-1 / 2}}{\sigma_{\varepsilon}}\right) \frac{H_{i, j}^{n-1 / 2} \hat{\varepsilon}_{i, j}^{n-1 / 2}-H_{i-1, j}^{n-1 / 2} \hat{\varepsilon}_{i-1, j}^{n-1 / 2}}{\Delta x_{i-1 / 2}}\right]
\end{aligned}
$$

The horizontal velocity-gradient production term $P_{h}$ can be discretized as:

$$
\left(P_{h}\right)_{i, j}^{n+1 / 2}=H_{i, j}^{n+1 / 2}\left(\hat{v}_{t}\right)_{i, j}^{n-1 / 2}\left[\begin{array}{l}
\left.2\left(\frac{U_{i+1 / 2, j}^{n+1 / 2}-U_{i-1 / 2, j}^{n+1 / 2}}{\Delta x_{i}}\right)^{2}+2\left(\frac{V_{i, j+1 / 2}^{n+1 / 2}-V_{i, j-1 / 2}^{n+1 / 2}}{\Delta y_{j}}\right)^{2}+\right] \\
\left(\frac{V_{i+1 / 2, j}^{n+1 / 2}-V_{i-1 / 2, j}^{n+1 / 2}}{\Delta x_{i}}+\frac{U_{i, j+1 / 2}^{n+1 / 2}-U_{i, j-1 / 2}^{n+1 / 2}}{\Delta y_{j}}\right)^{2}
\end{array}\right]
$$

335 According to the expression of Eq. (22b), the vertical velocity-gradient production term $P_{\varepsilon V}$ 336 (the difference form of $P_{k V}$ is the same as $P_{\varepsilon V}$ ) can be discretized as:

$337\left(P_{\varepsilon V}\right)_{i, j}^{n+1 / 2}=2464.16 C_{\mu}{ }^{2} C_{2 \varepsilon} \frac{\left\{\frac{f}{8}\left[\left(U_{i, j}^{n+1 / 2}\right)^{2}+\left(V_{i, j}^{n+1 / 2}\right)^{2}\right]\right\}^{2}}{H_{i, j}^{n+1 / 2}}$ 


\subsection{Boundary conditions}

339 To perform the staggered-grid difference method, ghost cells are typically imposed around the outmost computational domain. The boundary of all scalar variables, e.g., $\eta^{B}, \hat{k}^{B}$, and $\hat{\varepsilon}^{B}$

341 (where the superscript $B$ denotes the boundary) is set at the center of the ghost grid; the boundary of all vector variables, e.g., $P^{B}$ and $Q^{B}$, is located at the center of the adjacent surface between the ghost cell and the outermost computational grid [27]. The boundary conditions selected for this study mainly include open boundaries and no-slip boundaries.

Open boundary conditions are applied mainly to inflow and outflow. For the tests conducted, subcritical flow is the most frequent, hence the boundary conditions assumed include a specific flow rate assigned at the upstream boundary location; in addition, uniform water depth is applied as the downstream boundary condition. The inflow boundary condition can be expressed as follows:

$$
\eta_{1}^{\mathrm{B}}=\eta_{2}^{\mathrm{B}}, P_{3 / 2}^{\mathrm{B}}=P_{5 / 2}^{\mathrm{B}}, Q_{1}^{\mathrm{B}}=Q_{2}^{\mathrm{B}}, \hat{k}_{1}^{\mathrm{B}}=\hat{k}_{2}^{\mathrm{B}} \text { and } \hat{\varepsilon}_{1}^{\mathrm{B}}=\hat{\varepsilon}_{2}^{\mathrm{B}}
$$

352 The outflow boundary condition can be expressed follows:

$$
\eta_{n}^{\mathrm{B}}=\eta_{n-1}^{\mathrm{B}}, P_{n-1 / 2}^{\mathrm{B}}=P_{n-3 / 2}^{\mathrm{B}}, Q_{n}^{\mathrm{B}}=Q_{n-1}^{\mathrm{B}}, \hat{k}_{n}^{\mathrm{B}}=\hat{k}_{n-1}^{\mathrm{B}} \text { and } \hat{\varepsilon}_{n}^{\mathrm{B}}=\hat{\varepsilon}_{n-1}^{\mathrm{B}}
$$

354 No-slip boundary conditions are applied on the side where there is the solid-wall. The flux on 355 the solid boundary is zero, i.e., $P_{l+1 / 2}^{\mathrm{B}}=Q_{l+1 / 2}^{\mathrm{B}}=0$, where the subscript $l+1 / 2$ denotes the 356 adjacent surface between the water and solid-wall cells and $l$ denotes the corresponding center of the wall cell. The boundary conditions for $\hat{k}$ and $\hat{\varepsilon}$ at the location $l$ are also necessary. small, the shear stress and the turbulent production at $l$ can be balanced approximately with the wall shear stress and the dissipation at $l+1 / 2$, respectively [13]. The depth-averaged statistic characteristics in the small region near the wall are assumed to be analogous to the turbulent features in the core region near the bed. In other words, per Eqs. (8), (11), and (15), $\hat{\varepsilon}_{l}^{\mathrm{B}}$ can be expressed approximately as follows:

$$
\hat{\varepsilon}_{l}^{\mathrm{B}}=u_{\mathrm{w} *}{ }^{2} \frac{d U^{\prime}}{d y^{\prime}}=\frac{u_{\mathrm{W} *}^{3}}{\kappa \mathrm{S}}
$$

where $d U^{\prime} / d y^{\prime}$ is the depth-averaged velocity gradient in normal coordinates near the solid wall. Based on the specifications of $\hat{v}_{t}$ and Eq. (4), $\left(\hat{v}_{t}\right)_{l}^{\mathrm{B}}$ can be expressed as $\left(\hat{v}_{t}\right)_{l}^{\mathrm{B}}=\kappa s u_{\mathrm{W}^{*}}$; $\hat{k}_{l}^{\mathrm{B}}$ can be written as follows: 


$$
\hat{k}_{l}^{\mathrm{B}}=\frac{u_{\mathrm{W} *}^{2}}{\sqrt{C_{\mu}}}
$$

$u_{\mathrm{w} *}$ must satisfy the following equation [24]:

$$
\frac{U^{\prime}}{u_{\mathrm{W}^{*}}}=\left\{\begin{array}{l}
\frac{1}{\kappa} \ln \left(\frac{E u_{\mathrm{W}^{*}} y^{\prime}}{v_{k}}\right) \text { for hydraulically smooth wall } \\
\frac{1}{\kappa} \ln \left(\frac{30 y^{\prime}}{k_{s}}\right) \quad \text { for hydraulically rough wall }
\end{array}\right.
$$

372 where $E=0.9$ is the constant; $U^{\prime}$ is treated as a depth-averaged shear velocity at $l$; both $s$

373 and $y^{\prime}$ are approximated by half of the normal space step at $l$.

375 To secure stable numerical results, the Courant-Friedrichs-Lewy (CFL) number $\mathrm{Cr}$ was 376 considered as the stability criterion within the proposed model and to enable its incorporation, 377 the following double stability conditions were imposed for $\mathrm{Cr}$ as suggested by the literature 378 [24]:

$$
\begin{aligned}
& \mathrm{Cr}_{\text {flow }}=\Delta t \max \left\{\max \left[\left(\frac{|P| / H+\sqrt{g H}}{\Delta x}\right)_{i, j}\right], \max \left[\left(\frac{|Q| / H+\sqrt{g H}}{\Delta y}\right)_{i, j}\right]\right\} \leq 1 \\
& \mathrm{Cr}_{\text {turbulence }}=\Delta t \max \left\{\max \left[\left(\frac{2\left(\hat{v}_{t}+v_{k}\right)}{(\Delta x)^{2}}\right)_{i, j}\right], \max \left[\left(\frac{2\left(\hat{v}_{t}+v_{k}\right)}{(\Delta y)^{2}}\right)_{i, j}\right]\right\} \leq 1
\end{aligned}
$$

where $\Delta t$ is set as the minimum of the $\Delta t$ values calculated by using Eqs. (34a) and (34b).

\section{Model Validations}

To validate the model previously described to quantify complex turbulence, its performance has been verified against experimental turbulent flows obtained under various circumstances: 1) a uniform gravel bed, 2) a $90^{\circ}$ bend, and 3) a suddenly expanding section. Numerical results were then compared against measured datasets as well as the calculated values from the standard $\hat{k}-\hat{\varepsilon}$ model and other numerical schemes. To distinguish among the different models' results, " $P F$ " and " $R R F$ " are used to represent the model constructed by the proposed formula and by Rastogi and Rodi's formula, respectively.

\subsection{Turbulent flow in a straight channel with gravel bed}

391 To verify the accuracy of the proposed model in replicating bed roughness and Reynolds

392 number effects on the formation of turbulent features, a series of experiments on open-channel

393 flows over rough beds conducted by Wang et al [38], were used for comparison. The 
experiments were conducted in a straight flat glass flume $13.5 \mathrm{~m}$ long, $0.60 \mathrm{~m}$ wide, and 0.60 deep [38]. The roughness elements on the bottom of the flume were composed of gravel with median diameter $d_{50}$ ranging from 2 to $40 \mathrm{~mm}$. Throughout all measurements, the simultaneous high-frequency velocities in the middle of the flume were obtained with an acoustic Doppler velocimeter $(\mathrm{ADV})$.

According to the ensemble averages terms used in this study, velocity samples were gathered to determine the mean flows and turbulent fields in the system. The mean velocities in $x$-, $y$-, and $z$-directions were estimated as follows [24]:

$\bar{u}=\frac{1}{N} \sum_{i=1}^{N} u_{i}, \bar{v}=\frac{1}{N} \sum_{i=1}^{N} v_{i}, \bar{w}=\frac{1}{N} \sum_{i=1}^{N} w_{i}$

where $N$ is the number of samples. The root-mean-square (marked as r.m.s) values of the velocity fluctuations are defined by the sample standard deviation:

r.m.s. $u^{\prime}=\sqrt{\frac{\sum_{i=1}^{N} u_{i}{ }^{2}-N \bar{u}^{2}}{N-1}}$, r.m.s. $v^{\prime}=\sqrt{\frac{\sum_{i=1}^{N} v_{i}{ }^{2}-N \bar{v}^{2}}{N-1}}$, r.m.s. $w^{\prime}=\sqrt{\frac{\sum_{i=1}^{N} w_{i}{ }^{2}-N \bar{w}^{2}}{N-1}}$

The turbulent kinetic energy $k$ can be calculated from the above definitions as follows:

$k=\frac{1}{2}\left[\left(\text { r.m.s. } u^{\prime}\right)^{2}+\left(\text { r.m.s. } v^{\prime}\right)^{2}+\left(\text { r.m.s. } w^{\prime}\right)^{2}\right]$

The corresponding depth-averaged $\hat{k}$ can be obtained by depth-averaging the vertical profiles. The depth-averaged data calculated from vertical measured regions was taken to represent the entire depth at the corresponding horizontal coordinate due to the operation constraints of the ADV. The deviations between the depth-averaged velocities obtained by this method and those calculated by the logarithm profile in whole depth are around 5\% [24].

The calculating dimensions in the numerical experiments were $4.0 \mathrm{~m} \times 0.6 \mathrm{~m} \times 0.6 \mathrm{~m}$, which are consistent with the turbulent regime used by Wang et al. [38]. The roughness heights of the glass wall and the gravel bed were set to $k_{\mathrm{s}}=0.02 \mathrm{~mm}$ and $k_{\mathrm{s}}=d_{50}$, respectively [39]. $k_{\mathrm{s}}$ is the same in the glass bed as the wall. A uniform grid size $\Delta x=\Delta y=0.02 \mathrm{~m}$ and time step of $0.002 \mathrm{~s}$ were used in the computation. Eleven typical cases of different gravel $d_{50}$ and Reynolds numbers (Table 1) were analyzed. Considering the weight balance between the boundary conditions and fully turbulent development in regards to the effects of the turbulent structure in 
the numerical flume, the inflow unit volume flux and outflow water depth were set to $H_{0} U_{0}$ and $H_{0}$, respectively.

According to the measurement results, $\hat{k}$ was strongly dependent on the flow conditions when $k_{\mathrm{s}}$ was constant and it increased as $R e$ increased. It is demanding to make any definite conclusion regarding the effects of $k_{\mathrm{s}}$ on $\hat{k}$, however, because it is too difficult to keep Re or $\mathrm{Fr}$ neatly constant in any experiment. The ADV operation limitations and geometrical inhomogeneity over the gravel bed were likely to be responsible for this phenomenon.

The numerical results of the models derived from different expressions of $P_{k V}$ and $P_{\varepsilon V}$ are also presented in Table 1. The $P F$ values deviate slightly from the measured data, but there is reasonable agreement between them for the given ranges of $k_{\mathrm{s}}$ and $R e$. The $R R F$ results contained larger error than PF considering the whole range of results. The average of the error for all the test cases $\mathrm{C} 1$ to $\mathrm{C} 11$, for the $P F$ and $R R F$ models are, respectively, 49.67 and $108.45 \%$. With low bed roughness, $k_{\mathrm{s}}<5 \mathrm{~mm}$, both sets of results deviate gradually from the experimental values as $k_{\mathrm{s}}$ decreases. This behaviour can be attributed to the anisotropic tendency under which turbulent energy is redistributed over a smooth bed more slowly than over a rough one, which may be enhanced as roughness size decreases. Overall, the results show that the proposed model can effectively simulate turbulent flows over most of the gravel bed.

\subsection{Turbulence of open-channel flow in a $90^{\circ}$ bend}

Furthermore, the performance of numerical models $P F$ and $R R F$ and other models presented by Cea et al. [11] was compared against turbulent flows in open channel with a $90^{\circ}$ bend based on the experimental conditions described by Bonillo [40]. The experimental setup was identical to that described by Cea et al. [11], as shown in Fig. 3. The flow domain was made of two straight flat sections linked by a $90^{\circ}$ bend. The length and width of the first section were 4.835 $\mathrm{m}$ and $0.86 \mathrm{~m}$, respectively; those of the second section were $4.43 \mathrm{~m}$ and $0.72 \mathrm{~m}$, respectively. The bed of Segment 1 is $0.013 \mathrm{~m}$ higher than the bottom of Segment 2. The bottom and two sidewalls of the open channel are made of smooth concrete.

For calculation convenience and comparability among similar models, the flow region was discretized using a uniform grid of $\Delta x=\Delta y=0.02 \mathrm{~m}$. The Manning's coefficient of the bed surface selected was the same as Cea et al.'s [11] $0.016 \mathrm{~s} / \mathrm{m}^{1 / 3}$. The roughness height of the 
sidewall surface was assumed to be $k_{\mathrm{s}}=0.001 \mathrm{~m}$ [39]. The time step was fixed to $\Delta t=0.005 \mathrm{~s}$ to maintain numerical stability. The resulting $\hat{k}$ values at three different cross sections are shown in Fig. 4. The values calculated by two classical turbulence models [11], the $K E$ model and RSM model, are also included. Cea et al. [11] also developed a finite volume model to solve 2D SWEs, in which two famous turbulence models were used for comparison. The KE model is based on the assumption of isotropic eddy viscosity to solve the Reynolds stresses, while the RSM model directly solves each Reynolds stress without any restrictive hypothesis. Though the KE model equations are very similar to those of the RRF model, the location of $H$ in the diffusion term and the value of $\sigma_{\varepsilon}$ in the former differ from those of the latter.

As shown in Fig. 4, the PF results are very close to the experimental data in both the left sidewall area $(0 \leq x \leq 4.955)$ and right sidewall region $(5.195 \leq x \leq 5.555)$, where $B$ is the width of the models. In short, the flow features in both regions can be captured adequately by the PF model. In the intermediate region $(4.955 \leq x \leq 5.195)$, none of the numerical curves matched the measured data. This may be because the basic assumption of the shallow water equations is difficult to satisfy in the strong shear and bend zone, which includes intense 3D turbulence. The proposed model yielded accurate results overall despite some data scattering in the shear region.

\subsection{Turbulent characteristics in an expanding section}

An additional experiment was conducted to determine whether the proposed model can simulate the turbulent flows on an expanding channel [24]. The experiment was carried out in a flat expanding flume at the Hydraulic Engineering Laboratory at National University of Singapore. The length, width, and depth of the flume are respectively $15 \mathrm{~m}, 0.6 \mathrm{~m}$, and $0.6 \mathrm{~m}$. As shown in Fig. 5, the widths of the channel upstream and downstream of $x=0$ are $0.3 \mathrm{~m}$ and $0.6 \mathrm{~m}$, respectively. The steady flows were strictly controlled by two pumps and a tail gate during the experiment; boundary conditions were the discharge at upstream $\left(0.024 \mathrm{~m}^{3} / \mathrm{s}\right)$ and the flow depth at downstream $(0.015 \mathrm{~m})$, respectively. 137 regular points of the intersection were set between the longitudinal coordinates $x=-0.10,0.25,0.50,0.75,1.00,1.25,1.50,1.75$, $2.00,2.25,2.50,2.75$, and $3.00 \mathrm{~m}$ and the lateral coordinates $y=0.05,0.10,0.15,0.20,0.25$,

$4850.30,0.35,0.40,0.45,0.50$, and $0.55 \mathrm{~m}$ to observe velocity profiles and further inspect turbulence changes. 
instrument were monitored following procedure already published in literature [24]. Similar to the process in Section 3.1, the depth-averaged data $(U, V, \hat{k}$, and $\hat{\varepsilon})$ were further obtained.

We used identical numerical settings and discretization as Lin [24] to simulate turbulent flows in this study. The computational analysis was performed in a rectangular region of $9.0 \mathrm{~m} \times 0.6 \mathrm{~m}$ and differentiated by a $180 \times 30$ uniform grid with increments of $\Delta x=0.05 \mathrm{~m}$ and $\Delta y=0.02 \mathrm{~m}$; the length and width of the narrow channel upstream were $2.0 \mathrm{~m}$ and $0.3 \mathrm{~m}$, respectively. The upstream and downstream boundary conditions were unit volume flux of $0.08 \mathrm{~m}^{2} / \mathrm{s}$ and water depth of $0.15 \mathrm{~m}$, respectively. The Manning coefficient of the bed was $m=0.01 \mathrm{~s} / \mathrm{m}^{1 / 3}$ and the sidewall was considered to be smooth. The time step used was $\Delta t=0.001 \mathrm{~s}$.

The computed longitudinal velocities at different transverse sections were non-dimensionalized by the approach velocity $U_{0}$ and compared against the experimental results as shown in Fig. 6. In the upstream region without extension, i.e., $\mathrm{X}_{\mathrm{a}}=-0.1 \mathrm{~m}$, the $U$-velocity distribution was highly uniform. The sudden expansion in the profile of the sidewall induces strong non-uniformity to the velocity profiles, which was clearly observable per the flow separation and wake region in the detached flow. A recirculation zone (i.e., $0 \leq x / B \leq 8$ ), which was generated mainly due to the stagnant effect of the right sidewall of the building and the southern boundary of the channel, could also be clearly seen. In the downstream section, the longitudinal velocities gradually tended towards uniformity. By contrast, the maximum forward and backward velocities for both methods closely matched experimental data after the change in channel width, especially in the circulation region.

Similar to the $U$-velocity profiles, the non-dimensional transversal velocities calculated by the two models are shown in Fig. 7. In the main flow region (the area between $y=0.3$ and $0.6 \mathrm{~m}$ ), the computed results agree fairly well with experimental data. A steep velocity gradient occurred downstream of the building due to the strongly deflected flow. In the circulation zone (from $y=0.0$ to $0.3 \mathrm{~m}$ ), the velocity changes computed via $R R F$ method do not agree very well with the experimental data. The proposed method agrees slightly better with the experimental data but still underestimates the actual velocity by an average of about $50 \%$. In that region, the distribution of horizontal velocities is quite skewed over the full depth. The improved turbulence model does not sufficiently offset the defect of the hydrodynamic assumption in the deflected zone. 
Figure 8 shows a comparison among computed $\hat{k} / U_{0}^{2}$ values and experimental data. The proposed method yielded close agreement in the transverse section located $X_{\mathrm{a}}=-0.1 \mathrm{~m}$ upstream of the abrupt extension, in which the statistical turbulence features are similar to the turbulent characteristics in the open-channel uniform flow. The RRF results, by contrast, overestimated by about $10 \%$. At the upper-right corner of the building, where the flow separation began, sizable boundary irregularities were a source of turbulent energy in the horizontal direction. The horizontal bursts of turbulent activity propagated downstream and expanded on both sides, forcing the $\hat{k}$ values towards uniformity. Despite some scattering in the circulation zone, $P F$ yielded slightly more accurate results than $R R F$ overall.

The dimensionless profiles of turbulent dissipation $\hat{\varepsilon}$ calculated by the two models were compared against experiment data as shown in Fig. 9. In the main area and circulation zone, the $P F$ results were a better fit to the data than the $R R F$ results. In the transitional region between them, especially near the corner of the building, high levels of energy dissipation were produced due to large-scale eddies and a steep velocity gradient. The proposed method better reflected this phenomenon than the $R R F$, but still overestimated it by around $80 \%$. The difference is mainly attributable to experimental error due to ADV operation limitations and partly by the strong $3 \mathrm{D}$ flow structure in corresponding regions. These results altogether indicate that $P F$ can better describe the hydraulic and turbulent structure in the abrupt expanding channel than $R R F$.

\section{Turbulent Flows near Two Groins in a Natural Waterway}

To further investigate the performance of the model developed and illustrate its engineering application, it was used to compute complex turbulent flows and the correspondent navigation conditions in natural waterways and waterways after the construction of two buildings.

\subsection{Site description and numerical setup}

The Yangliu moraine is located in a relatively straight gorge on the upper reach of the Yangtze River, approximately $1017.8 \mathrm{~km}$ upstream of Yichang City, a prefecture-level city in Hubei Province, China. The length and width of the moraine are respectively $1800 \mathrm{~m}$ and $400 \mathrm{~m}$, as shown in Fig. 10. The top level of the moraine near the mid-channel, where the highest point is $5 \mathrm{~m}$ above the designed water level, is higher than the shoreline. To this effect, the unique bed structure forms a concave basin. The water level in the river must be at least $1 \mathrm{~m}$ higher than the designed level to satisfy the necessary flow conditions. Under the restriction of the moraine, the main channel was squeezed to only $250 \mathrm{~m}$ in width. Another upstream moraine section of the main reach, the Huangjia moraine, is affected by a shorter lateral flow area with $300 \mathrm{~m}$. A 
transitional shallow zone (width $500 \mathrm{~m}$ ) was formed between the two moraines.

560 The transitional region, in which the flow is relatively slow, has a flat and straight geometric

561 bed. There is no erosion of river bed material along the ship route during the dry seasons due

562 to hydrodynamic limitations, hence there is only a small water depth for navigation during this

563 period. The minimum depths were recorded in 1993 and 2006, $2.3 \mathrm{~m}$ and $2.5 \mathrm{~m}$, respectively.

564 Under these hydraulic conditions, it is very challenging to satisfy the class III navigation

565 conditions necessary for free travel of ships with dimensions $560 \mathrm{~m} \times 50 \mathrm{~m} \times 2.7 \mathrm{~m}$.

567 To guarantee class III navigational standards throughout the entire year, the local waterway 568 bureau constructed two groins on the left side of the shallow region in 2008 (Fig. 10). Groin 1

569 was built at the upstream reach with a $60^{\circ}$ angle between its axis and the main stream for 570 smooth flow transition. Groin 2 was constructed at the downstream end with a corresponding 571 angle of $75^{\circ}$. The length of the two groins are respectively $281 \mathrm{~m}$ and $313 \mathrm{~m}$; both of their 572 hook heads are $87 \mathrm{~m}$. The local waterway bureau measured the bed topography of the reach, the water surface elevation on the shipping route, and the flow velocity in three streamlines on January $15^{\text {th }}, 2010$, to investigate the effects of the two groins on the waterway (Fig. 10) and the consequent riverbed erosion. For this specific date, the total discharge recorded at the upstream section was $3120 \mathrm{~m}^{3} / \mathrm{s}$ and the average water level at the downstream section was $253.44 \mathrm{~m}$, values derived by a nearby hydrological station and a water gauge, respectively.

According to the geological analysis conducted to characterise the properties of the soil in the two boreholes (N1 and N2, as shown in Fig. 10), the bed materials of the transitional shoal region are mainly composed of boulders, sand-cobbles, and brick red sandstones. The mixture of boulders and sand-cobbles typically forms a covering layer $2.3 \mathrm{~m}$ thick above the base layer composed by sandstones. The maximum boulder particle-size is $0.9 \mathrm{~m}$ and the mass percentage of sand-cobble with 0.03-0.2 m diameter is about $65 \%$. The sieving results at N1 and N2 indicate that $d_{50}$ in the reach is approximately $0.04 \mathrm{~m}$.

589 The river section that was simulated within the model developed to verify its applicability is

$5901964 \mathrm{~m}$ long and $924 \mathrm{~m}$ wide, based on the topographic features of Yangliu and Huangia 591 moraines (Fig. 10). The region that was computed was discretized by applying a uniform 592 Cartesian grid size with dimensions $\Delta x=\Delta y=4 \mathrm{~m}$. The median particle size of the bed 
material was set at $d_{50}=0.04 \mathrm{~m}$ and the time step was set at $\Delta t=0.002 \mathrm{~s}$. A flow rate of 3120 $\mathrm{m}^{3} / \mathrm{s}$ and an averaged water surface elevation of $253.44 \mathrm{~m}$ were respectively assigned to the inflow and outflow boundaries according to the simplified methods presented by Zhang [41].

\subsection{Results and performance}

597 Figure 11 shows the comparisons between observed and simulated water levels along the ship 598 route. The average discrepancy between observed data and $P F$ results is $0.124 \mathrm{~m}$, while the 599 discrepancy between observed data and $R R F$ results is $0.127 \mathrm{~m}$. Although $P F$ slightly 600 outperforms $R R F$, especially in the transition section, both have discrepancies from the 601 measured data. This is mainly due to the uniform roughness coefficient that was imposed in the 602 whole river, which does not replicate accurately the natural variety of roughness along the 603 system. In fact, the bed material is uneven in both longitudinal and transverse directions and 604 the bed structure is irregular in such a natural channel hence the bed resistance status could not 605 be characterised by a uniform median size as assumed in this study. Therefore, there is huge 606 potential to greatly improve the results if $d_{50}$ was obtained at each specific site for the 607 numerical calculations.

608

609 The flow velocity along three different streamlines was computed and compared against the 610 measured values as shown in Fig. 12. Overall, the calculated curves agree well with the 611 experimental data except in the transition region of Streamline 2, where the maximum absolute 612 deviation was about $0.3 \mathrm{~m} / \mathrm{s}$. Similar to the measured water surface elevations, the surveyed 613 points of Streamline 2 were mainly distributed on the route. The $d_{50}$ in the transitional area 614 was generally underestimated. Although there were no significant differences between the two 615 numerical results at most points, $P F$ was more accurate than $R R F$ near the two groins (the groins 616 are approximately at $x=900$ and $x=1200 \mathrm{~m}, P F$ model is more accurate between $x \sim 800-1300$

$617 \mathrm{~m})$. Because the turbulence parameters were not measured, we only depict the numerical $\hat{k}$ at 618 two cross sections symbolically to reflect the relevant turbulence characteristics (Fig. 13). The 619 two sections, A-A and B-B (displayed as dash lines in Fig. 10 and their results presented in Fig. 620 13), are located downstream of the two groins where the longitudinal distance to the root of the 621 groin is approximately equal to the length of the corresponding groin (Fig. 10). As shown in 622 Fig. 13, the numerical values of $R R F$ are about 2 times the $P F$ results in the mainstream area 623 and approximately 1.5 times $P F$ 's in the circulation zone behind the groin. Furthermore, for 624 each method, the numerical values at B-B were larger than those at A-A.

626 To further explore the navigation scenario discussed above, we calculated the water depth by 627 the proposed model as shown in Fig. 14. In the transitional region of a straight waterway, the 
water depth was at least $3.5 \mathrm{~m}$ and the width of the water surface was greater than $80 \mathrm{~m}$. To this effect, the river would satisfy category III navigation conditions during the dry season. Our results altogether showed that flow behavior in a natural river can indeed be captured with a reasonable accuracy by the proposed method.

\section{Conclusions}

This study focused on developing a second-order numerical scheme of spatial staggered-grid difference and temporal leap-frog discretization for simulating the turbulent flows on a complex roughness bed. The model is based on the 2D SWE model and the $\hat{k}-\hat{\varepsilon}$ turbulent closure model. The depth-averaged vertical turbulent generation from the universal notions of the Kolmogorov $-5 / 3$ spectrum and the log-law was derived and used as a substitute for the vertical velocity gradient term of the standard $\hat{k}-\hat{\varepsilon}$ model. The proposed $\hat{k}-\hat{\varepsilon}$ model reflects the vertical turbulent production of various scales more robustly than the standard model.

Extensive comparisons were conducted against complex turbulent flows between the proposed model, standard $\hat{k}-\hat{\varepsilon}$ model, and measurement data. The proposed method effectively captured the experimental hydrodynamic features, especially the turbulence characteristics, and improved the simulation accuracy over the standard $\hat{k}-\hat{\varepsilon}$ model. The proposed model also showed better global performance than other 2D shallow numerical schemes.

Furthermore, the turbulent flows over the roughness bed in a natural river were simulated and analyzed. Results showed that the depth-averaged turbulent energy increases as Reynolds number increases when the roughness height is constant. Additionally, the numerical results for Yangliu moraines reach showed that the river navigation conditions are satisfied after constructing the two groins under the flow conditions we considered.

3D simulations will even more adequately disclose the fundamental physics of flows. In this present model application, a large flow domain (Yangliu moraines reach) was considered hence fully $3 \mathrm{D}$ computations would have not been very cost-effective. By using the proposed 2D Shallow Water Equations with improved turbulence modelling techniques, it was possible to achieve reasonably engineering accuracy but with a much lower CPU cost. Despite that, considering that $3 \mathrm{D}$ analysis would provide a more accurate predictive tool for the flow problems and promote a deeper understanding of the physics of the fluid motion, future work will target the development of an enhanced quasi-three-dimensional shallow flow solver. 
662 Per the large amplitude and gradient of turbulent energy near the structures, local scouring may

663 occur in rainy seasons and gradually alter the bottom configuration, affecting the stability of

664 the groins. To safeguard the regulation buildings, an effective sediment transportation model is

665 necessary to investigate the bed deformation and future research should incorporate more cases

666 to replicate accurately a variety of scenarios to achieve a universal model that can be reliable

667 for each situation.

\section{Acknowledgements}

669 Dr. Xiekang Wang and Dr. Xianye Wang kindly provided the 3D experimental data in Section

670 3.1. The authors are also grateful to Dr. Luis Cea for the data in Section 3.2.

\section{$671 \quad$ Funding}

672 This work was supported by the National Key Research and Development Program of China

673 (Grant No: 2016YFE0122500, 2013CB036401 and 2013CB036402), China Postdoctoral

674 Science Foundation (Grant No: 2016M591184) and Programme of Introducing Talents of

675 Discipline to Universities (Grant No: BC2018038).

\section{Notation}

$677 \quad f \quad=$ bed friction factor $(-)$

$678 g \quad=$ gravitational acceleration $\left(\mathrm{ms}^{-2}\right)$

$679 \quad H \quad=$ water depth $(\mathrm{m})$

$680 \quad k \quad=$ turbulent energy $\left(\mathrm{m}^{2} \mathrm{~s}^{-2}\right)$

$681 \hat{k} \quad=$ depth-averaged turbulent energy $\left(\mathrm{m}^{2} \mathrm{~s}^{-2}\right)$

$682 \quad k_{s} \quad=$ equivalent sand roughness $(\mathrm{m})$

$683 \quad l_{m} \quad=$ length scale of turbulent flow $(\mathrm{m})$

$684 m \quad=$ Manning's roughness coefficient $\left(\mathrm{sm}^{-1 / 3}\right)$

$685 P, Q \quad=$ unit volume flux in $x$ - and $y$-directions, respectively $\left(\mathrm{m}^{2} \mathrm{~s}^{-1}\right)$

$686 \quad R \quad=$ hydraulic radius $(\mathrm{m})$

687 Re $\quad=$ Reynolds number $(-)$

$688 T_{x x}, T_{y y} \quad=$ depth-averaged normal stress in $x$ - and $y$-directions, respectively $(\mathrm{Pa})$

$689 T_{y x}, T_{x y} \quad=$ depth-averaged shear stress in $x$ - and $y$-directions, respectively $(\mathrm{Pa})$

$690 \quad u^{*} \quad=$ velocity scale of turbulent flow $\left(\mathrm{ms}^{-1}\right)$

$691 \quad u_{*} \quad=$ friction velocity $\left(\mathrm{ms}^{-1}\right)$

$692 U, V, W=$ depth-averaged velocity in $x-, y-$ and $z$-directions, respectively $\left(\mathrm{ms}^{-1}\right)$ 


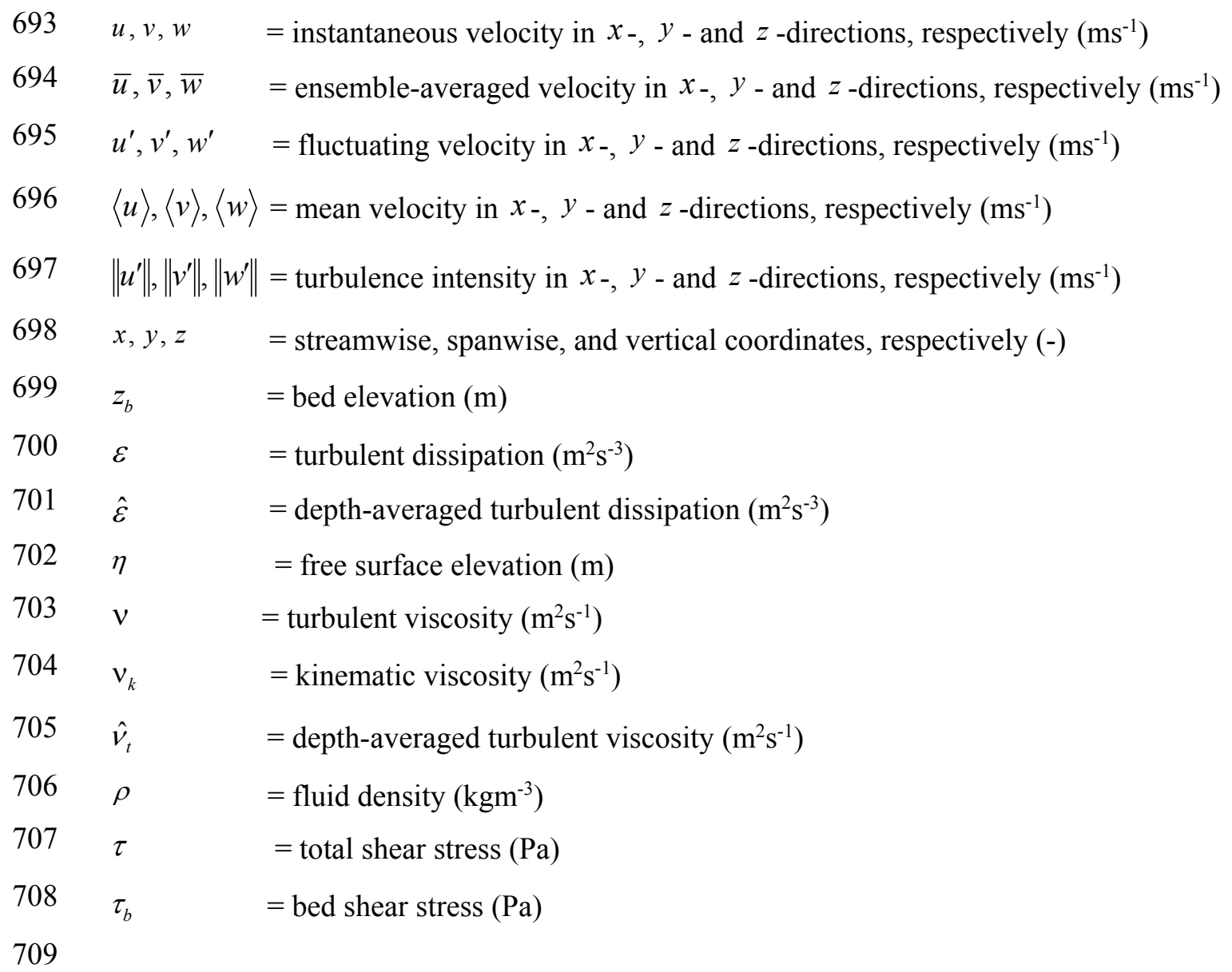

\section{References}

1. Rodi, W., Mansour, N. N. (1993), Low Reynolds number $k-\varepsilon$ modelling with the aid of direct simulation data, J Fluid Mech 250, 509-529.

2. Pope, S. B. (2000), Turbulent Flows, Abingdon, Routledge.

3. Bhaganagar, K., Kim, J., Coleman, G. (2004), Effect of roughness on wall-bounded turbulence, Flow, Turbulence and Combustion, 72, 463-492.

4. Hinterberger, C., Fröhlich, J., Rodi, W. (2008), 2D and 3D turbulent fluctuations in open channel flow with $\mathrm{Re}_{\tau}=590$ studied by large eddy simulation, Flow, Turbulence and Combustion, 80, 225-253.

5. Erpicum, S., Meile, T., Dewals, T. M., Pirotton, M., Schleiss, A. J. (2009), 2D numerical flow modeling in a macro-rough channel, Int J Numer Meth Fluids, 61, 1227-1246.

6. Younus, M., Chaudhry, M. H. (1994), A depth-averaged $\hat{k}-\hat{\varepsilon}$ turbulence model for the computation of free-surface flow, Journal of Hydraulic Research, 32(3), 415-444.

7. Cao, Z. X., Hu, P. H., Hu, K. H., Pender, G., Liu, Q. H. (2014), Modelling roll waves with shallow water equations and turbulent closure, Journal of Hydraulic Research, 53(2), 161177. 
8. Pu, J. H., Shao, S. D., Huang, Y. F. (2014), Numerical and experimental turbulence studies on shallow open channel flows, Journal of Hydro-environment Research, 8, 9-19.

9. Campomaggiore, F., Cristo, C. D., Iervolino, M., Vacca, A. (2016), Inlet effects on rollwave development in shallow turbulent open-channel flows, J Hydrol Hydromech, 64(1), $45-55$.

10. Duan, J. G. (2004), Simulation of flow and mass dispersion in meandering channels, Journal of Hydraulic Engineering, 130(10), 964-976.

11. Cea, L., Puertas, J., Gázquez-Cendón, M. E. (2007), Depth averaged modelling of turbulent shallow water flow with wet-dry fronts, Arch Comput Methods Eng, 14, 303341.

12. Rastogi, A. K., Rodi, W. (1978), Predictions of heat and mass transfer in open channels, J Hydr Div, 104(3), 397-420.

13. Launder, B. E., Spalding, D. B. (1974), The numerical computation of turbulent flows, Computer Methods in Applied Mechanics and Engineering, 3, 269-289.

14. Wu, W. M., Wang, P. Y., Chiba, N. (2004), Comparison of five depth-averaged 2-D turbulence models for river flows, Archives of Hydro-Engineering and Environmental Mechanics, 51(2), 183-200.

15. Chen, Y. S., Kim, S. M. (1987), Computation of turbulent flows using an extended $k-\varepsilon$ turbulence closure model, CR-179204, NASA, pp21.

16. Booij, R. (1989), Depth-averaged $k-\varepsilon$ modelling, In Proc 23rd IAHR Congress, Delft, The Netherlands, Vol A, IAHR, pp. 198-206.

17. Babarutsi, S., Chu, V. H. (1991), A two-length-scale model for quasi two-dimensional turbulent shear flows, In Proc 24th Congress of IAHR, Madrid, Spain, Vol C, IAHR, pp $51-60$.

18. Yakhot, V., Orszag, S. A., Thangam, S., Gatski, T. B., Speziale, C. G. (1992), Development of turbulence models for shear flows by a double expansion technique, Phys Fluids A, Fluid Dynamics, 4(9).

19. Richardson, L. F. (1922), Weather Prediction by Numerical Process. Cambridge: Cambridge University Press.

20. Nezu, I., Nakagawa, H. (1993), Turbulence in Open-channel Flows, A. A. Balkema, Rotterdam.

21. Hunt, J. C. R., Eames, I., Westerweel, J., Davidson, P. A., Voropayev, S., Fernando, J., Braza, M. (2010), Thin shear layers - The key to turbulence structure, Journal of Hydroenvironment Research, 4, 75-82.

22. Kolmogorov, A. N. (1941), The local structure of turbulence in incompressible viscous fluid for very large Reynolds number, CR Acad Sci URSS, 30, 301-305. 
23. Cho, Y. S. (1995), Numerical Simulations of Tsunami Propagation and Run-up, PhD dissertation, Cornell University, Ithaca, NY.

24. Lin, Q. H. (2009), Numerical Simulation of Sediment Transport and Morphological Evolution, $\mathrm{PhD}$ dissertation, National University of Singapore, Singapore.

25. Lin, P. Z., Wu, Y. N., Bai, J. L., Lin, Q. H. (2011), A numerical study of dam-break flow and sediment transport from a quake lake, Journal of Earthquake and Tsunami, 5(5), 401428.

26. Zhang, Y. (2015), Study of Numerical Model for Simulating Evolution of Dam-break Flows, PhD dissertation, Sichuan University, China.

27. Zhang, Y., Lin, P. Z. (2016), An improved SWE model for simulation of dam-break flows, Proceedings of the Institution Civil Engineers - Water Management, 169(6), 260-274.

28. Idelchik, I. E. (1994), Handbook of Hydraulic Resistance, CRC Press, 3rd ed. Boca Raton, USA.

29. Abbaspour, A., Kia, S. H. (2014), Numerical investigation of turbulent open channel flow with semi-cylindrical rough beds, Journal of Civil Engineering, 18(7), 2252-2260.

30. Pu J. H., Wei J., Huang Y. (2017), Velocity distribution and 3D turbulence characteristic analysis for flow over water-worked rough bed, Water, 9(9), 668; doi:10.3390/w9090668.

31. Coles, D. (1956), The law of the wake in the turbulent boundary layer, J Fluid Mech, 1, 191-226.

32. Pu J. H., Tait S., Guo Y., Huang Y., Hanmaiahgari P. R. (2018), Dominant features in three-dimensional turbulence structure: comparison of non-uniform accelerating and decelerating flows, Environmental Fluid Mechanics, 18(2), pp.395-416.

33. Nakagawa, H., Nezu, I., Ueda, H. (1975), Turbulence of open channel flow over smooth and rough beds, Proc of Japan Soc Civil Engrs, 241, 155-168.

34. Jobson, H. E., Sayre, W. W. (1970), Vertical transfer in open channel flow, J Fluid Mech, $122,295-314$.

35. Ueda, H., Moller, R., Komori, S., Mizushina, T. (1977), Eddy diffusivity near the free surface of open channel flow, Int J Heat and Mass Transfer, 20, 1127-1136.

36. Hussain, A. K. M. F., Reynolds, W. C. (1975), Measurements in fully developed turbulent channel flow, J Fluids Eng, 97, 568-580.

37. Nezu, I., Rodi, W. (1986), Open-channel flow measurements with a laser Doppler anemometer, J Hydraulic Eng, 112, 335-355.

38. Wang, X. Y., Yang, Q. Y., Lu, W. Z., Wang, X. K. (2012), Experimental study of nearwall turbulent characteristics in an open-channel with gravel bed using an acoustic Doppler velocimeter, Exp Fluids, 52, 85-94. 

803

804
39. Chanson, H. (2004), The Hydraulics of Open Channel Flow: An Introduction, Butterworth-Heinemann, Oxford, UK.

40. Bonillo, J. (2000), Un modelo de transporte de sustancias solubles para flujos trubulentos en lamina libre. Tesis doctoral, Área de Ingeniería Hidráulica, Universidad de A Coruña.

41. Zhang, Y. (2011), Numerical Simulation of Flow around Groyne with the Different Pick Angles in the Inland Waterways, Master Thesis, Sichuan University, China. 


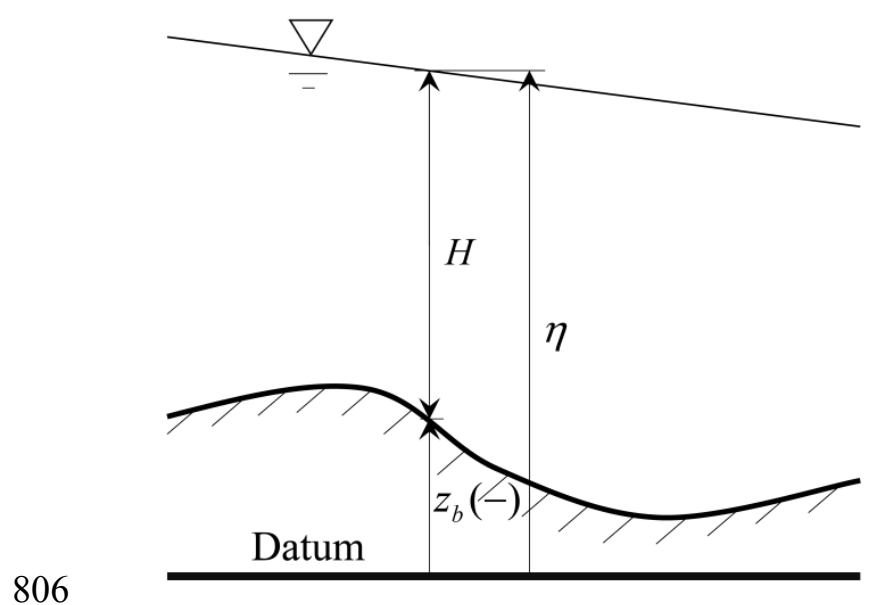

807 Figure 1 - Scheme illustrating an example of irregular bed elevation and free surface water 808 depth.

809 


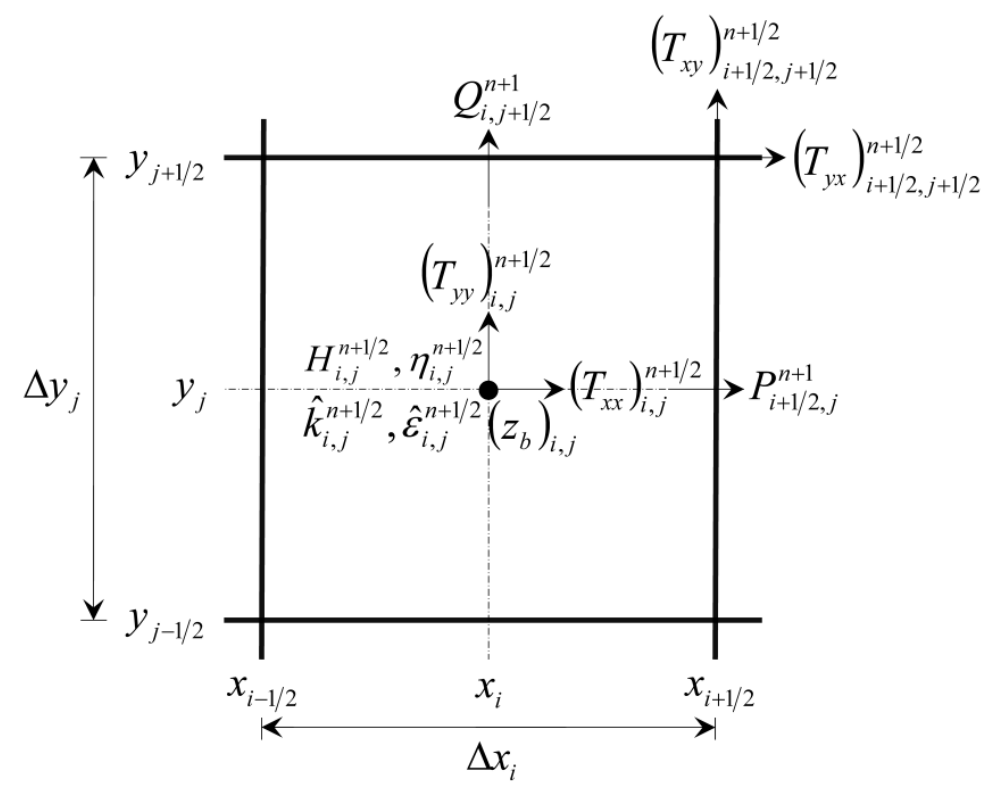

812 Figure 2 - Scheme of staggered grid which includes the locations of the variables considered in 813 a single cell.

814 


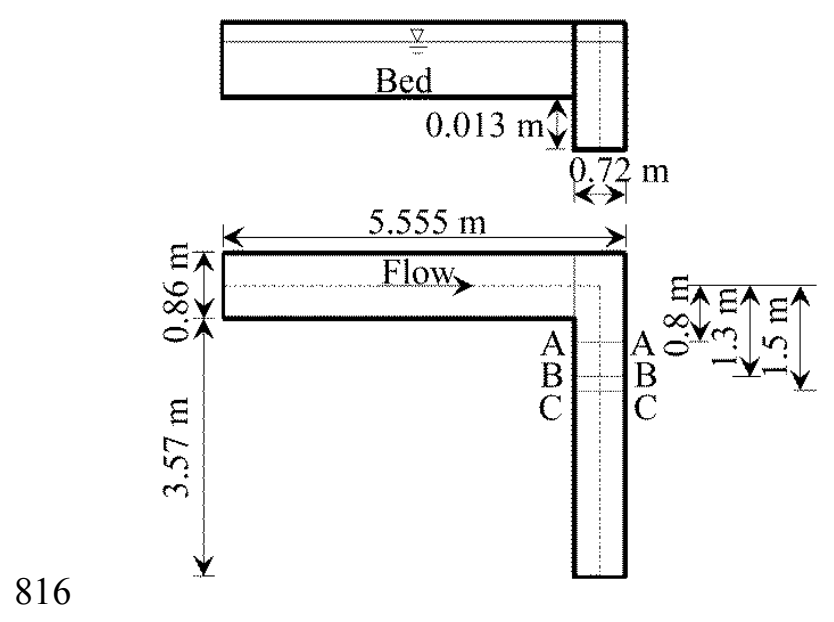

817 Figure 3 - Scheme of the experimental channel with a $90^{\circ}$ bend: front view (top) and top view 818 (bottom).

819 

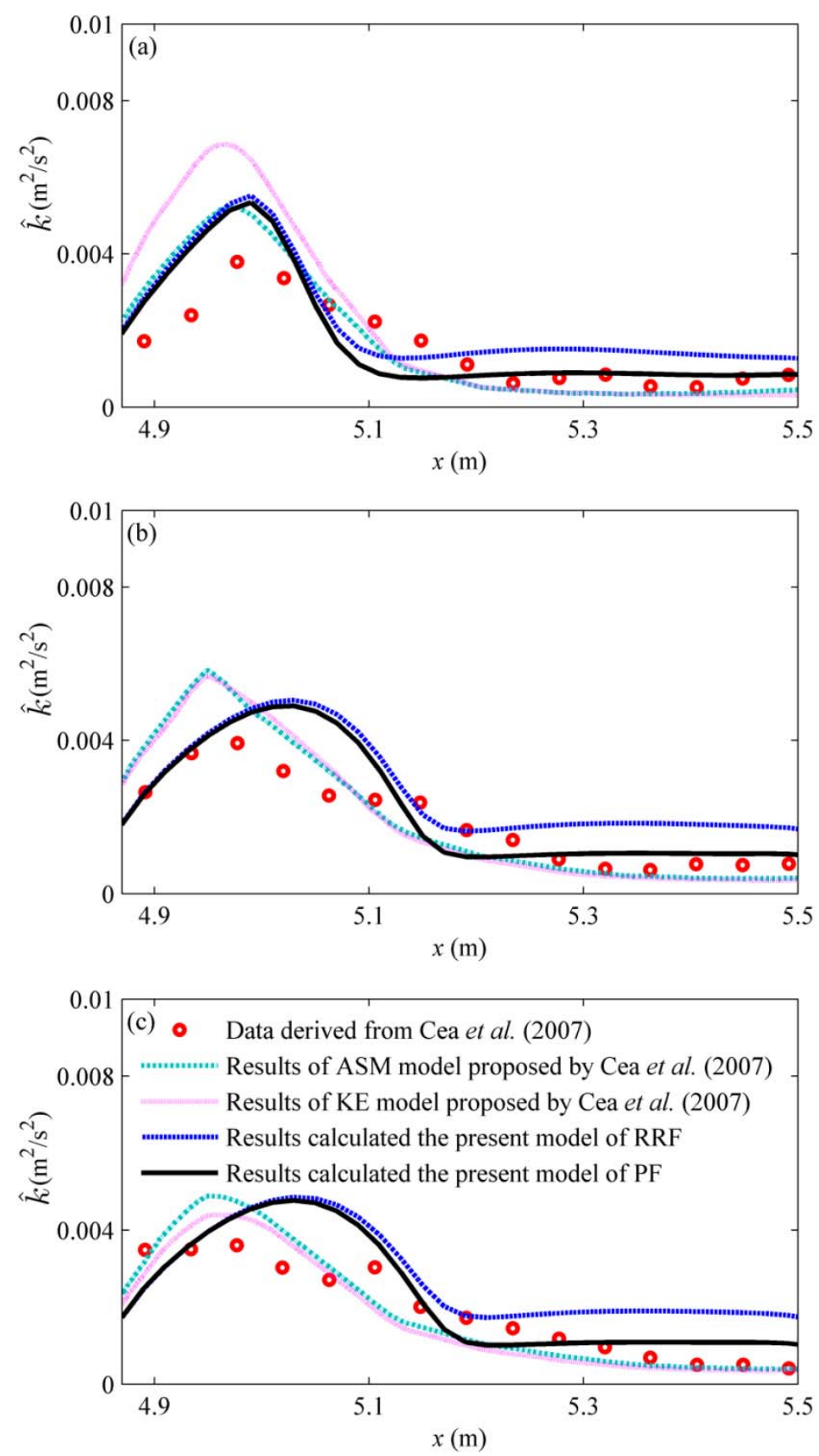

822 Figure 4 - Comparison of turbulent kinetic energy measured experimentally and calculated 823 numerically at three sections (a) A-A, (b) B-B and (c) C-C. 


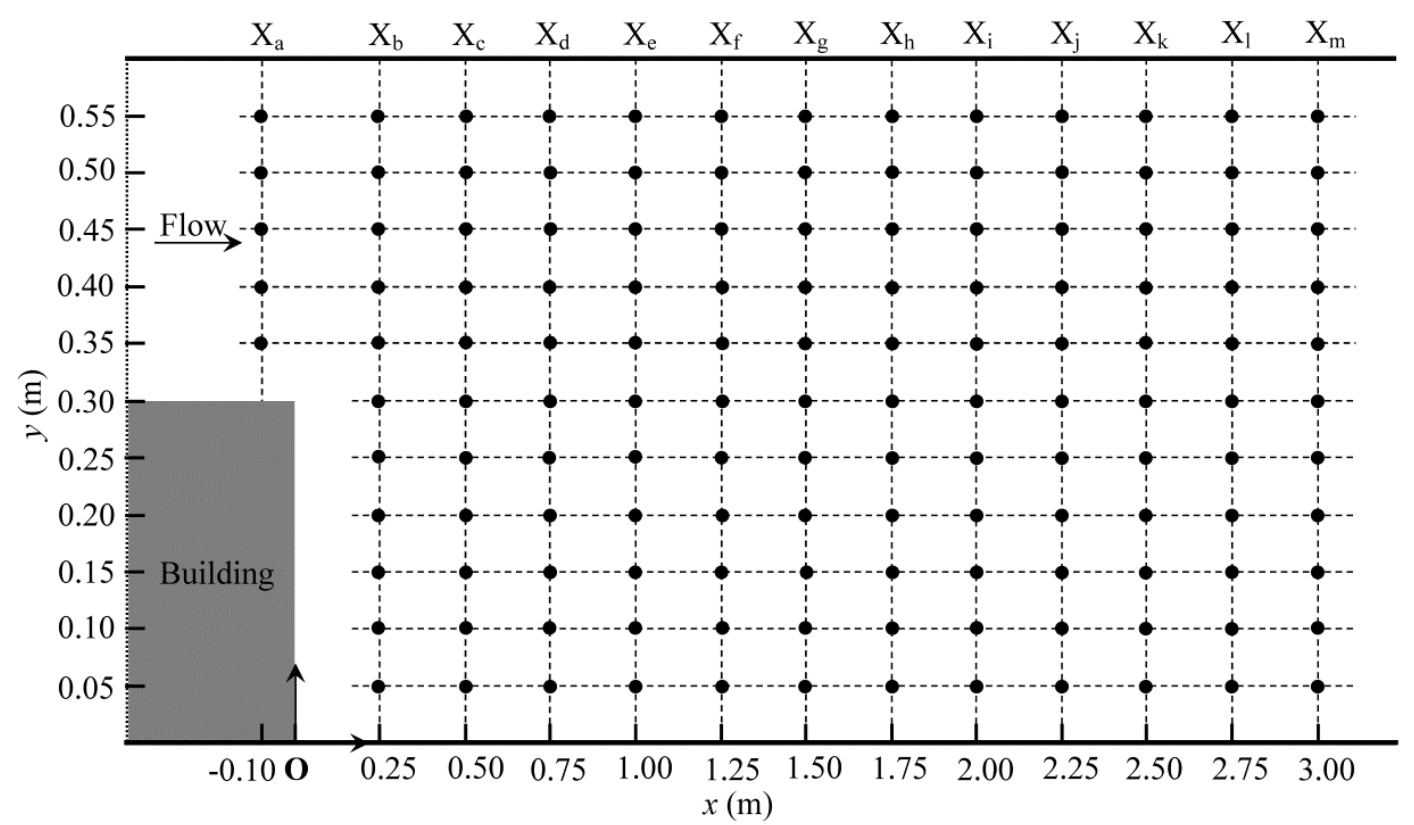

826

827 Figure 5 - Plan view of flume with an expanding section and the observed points.

828 


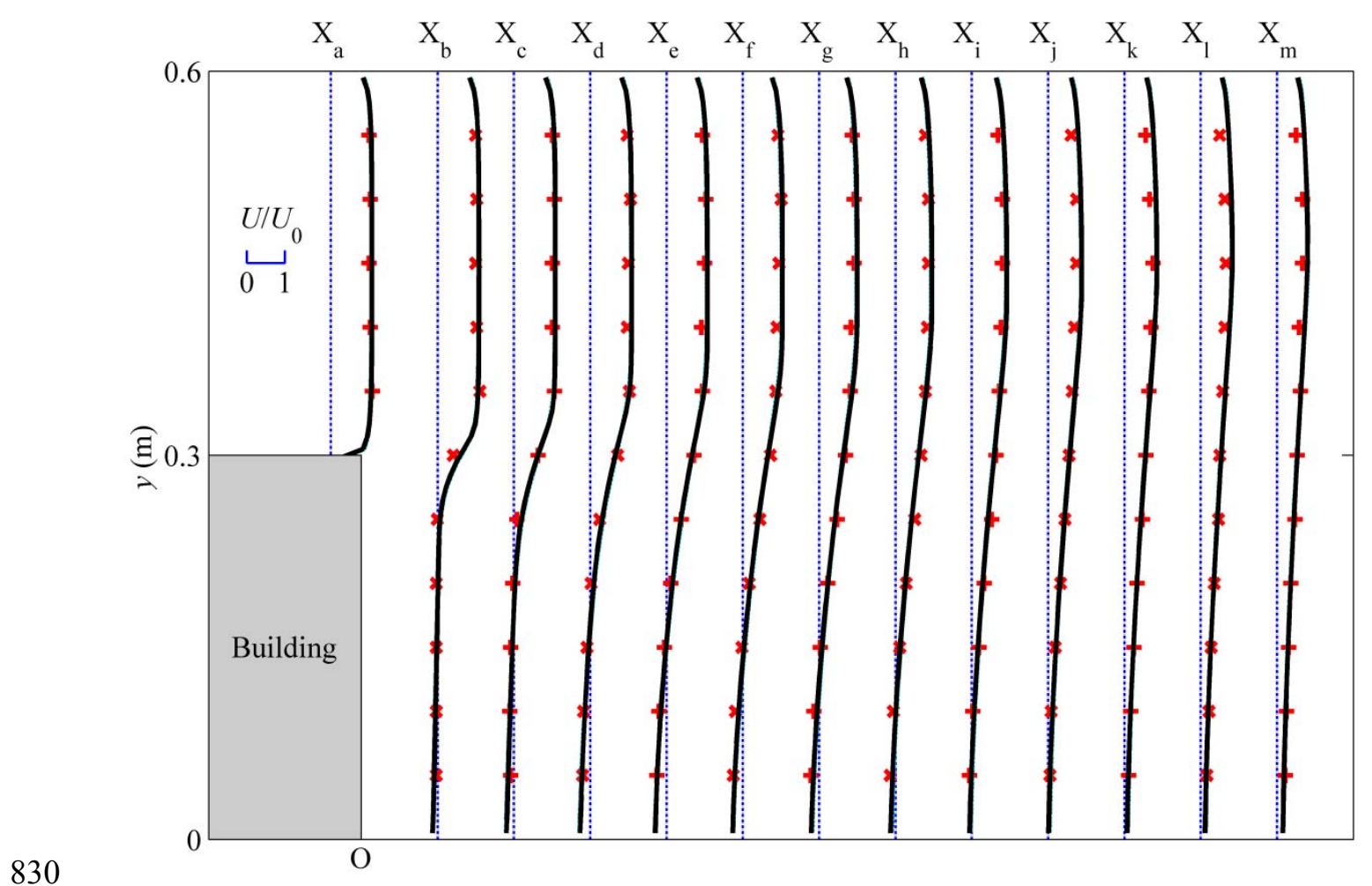

831 Figure 6 - Comparisons of non-dimensionalized $U$ between numerical results and experimental 832 data (Red crosses: experimental data; Dashed green line: RRF's results; Solid black line: PF's 833 results).

834 


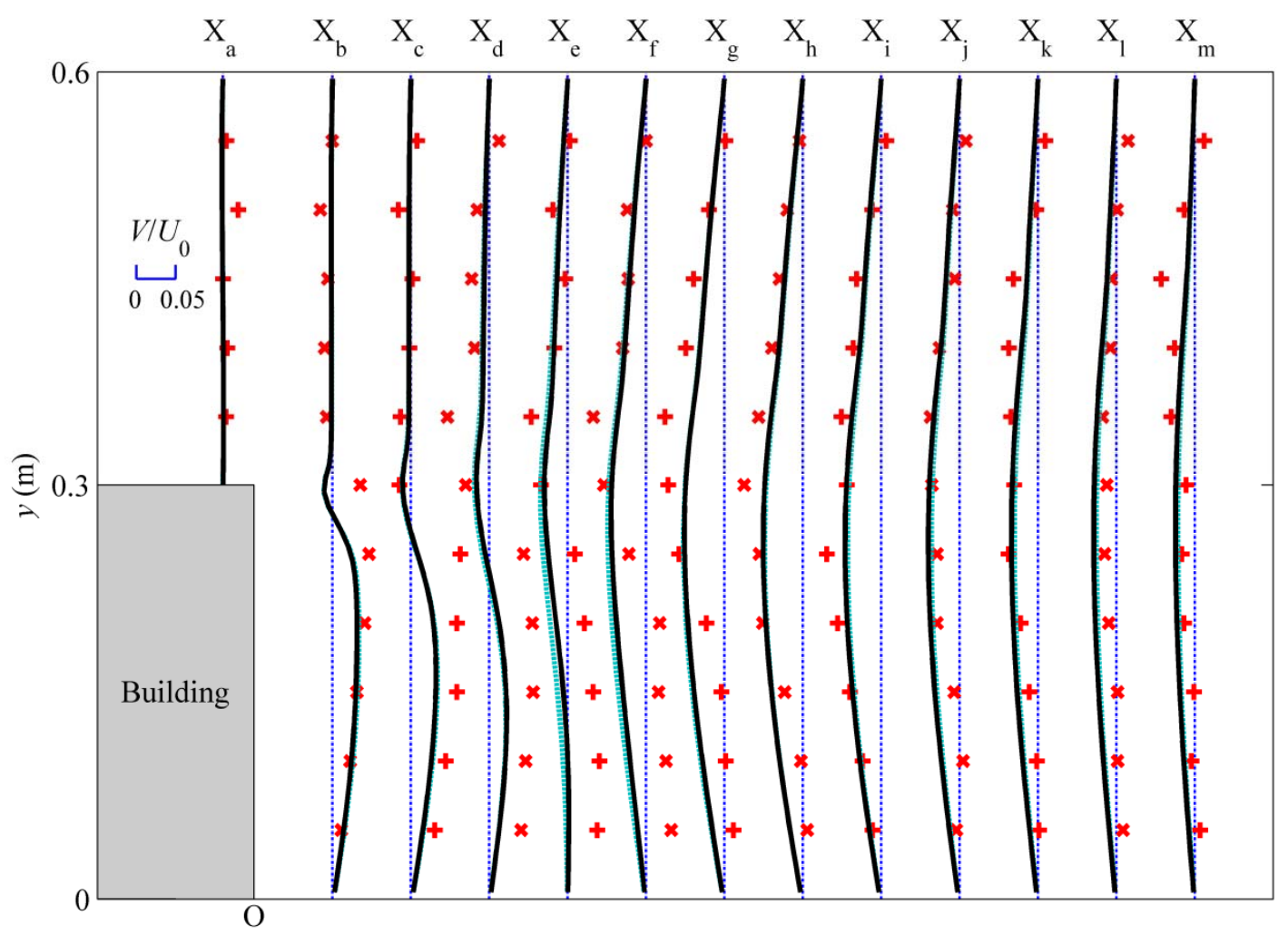

837 Figure 7 - Comparisons of non-dimensionalized $V$ between numerical results and experimental 838 data (Red crosses: experimental data; Dashed green line: RRF's results; Solid black line: PF's 839 results). 


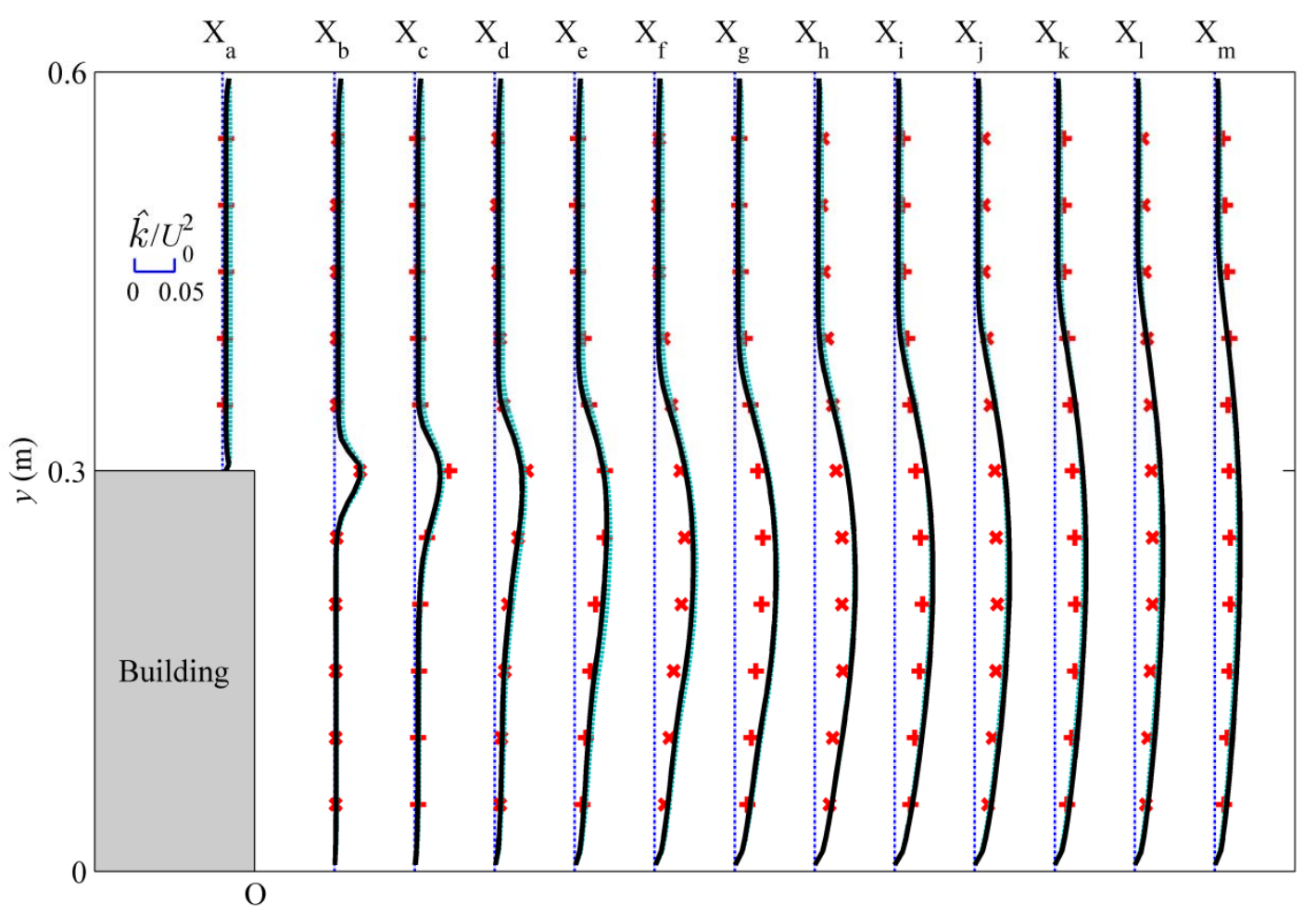

843 Figure 8 - Comparisons of non-dimensionalized $\hat{k}$ between numerical results and experimental 844 data (Red crosses: experimental data; Dashed green line: RRF's results; Solid black line: PF's 845 results). 


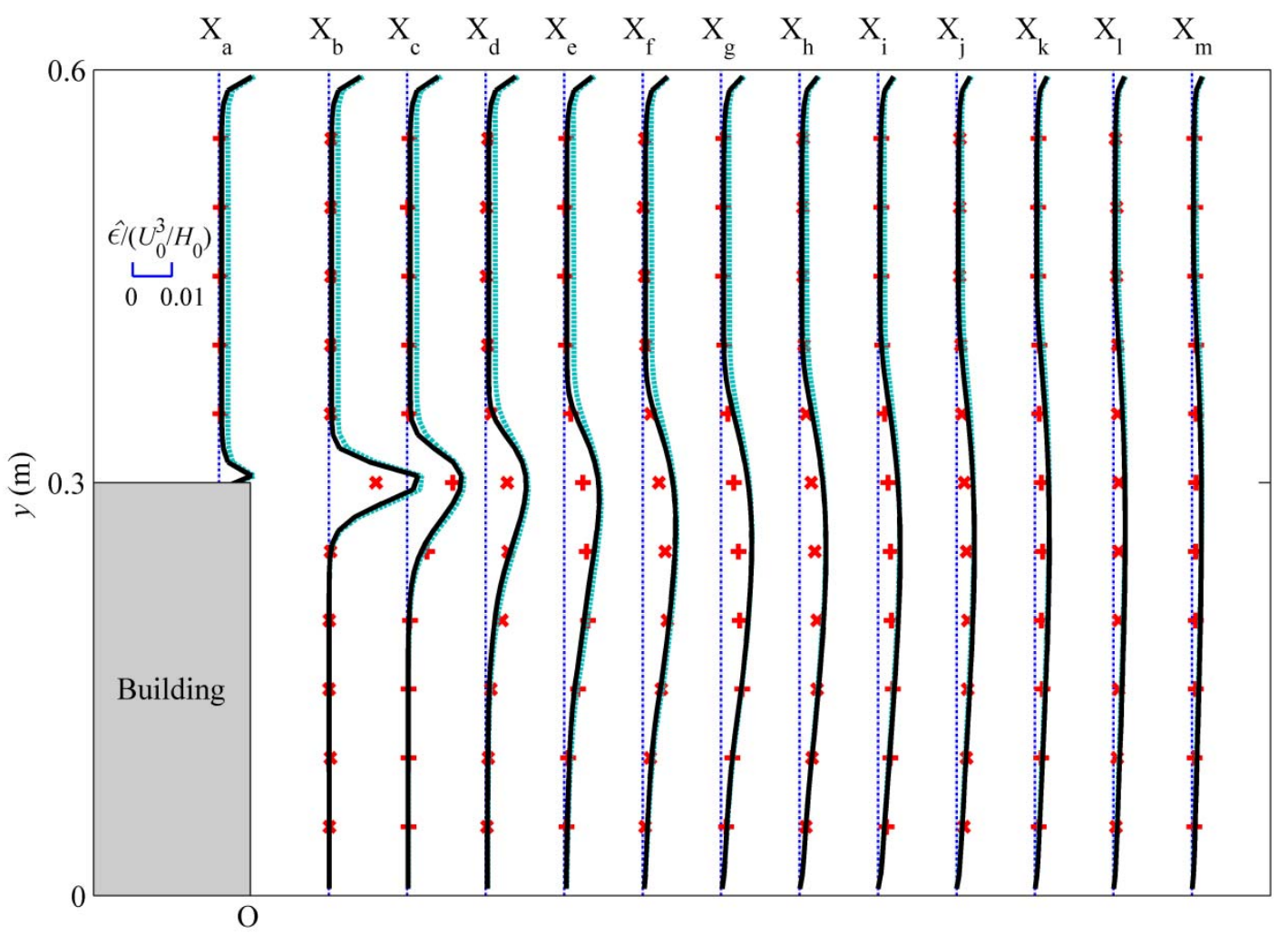

849 Figure 9 - Comparisons of non-dimensionalized $\hat{\varepsilon}$ between numerical results and experimental 850 data (Red crosses: experimental data; Dashed green line: RRF's results; Solid black line: PF's 851 results). 
(2) Topography

- Observation points of water level on the ship route

- Survey points of velocity in the streamline 1

4 Survey points of velocity in the streamline 2

v Survey points of velocity in the streamline 3
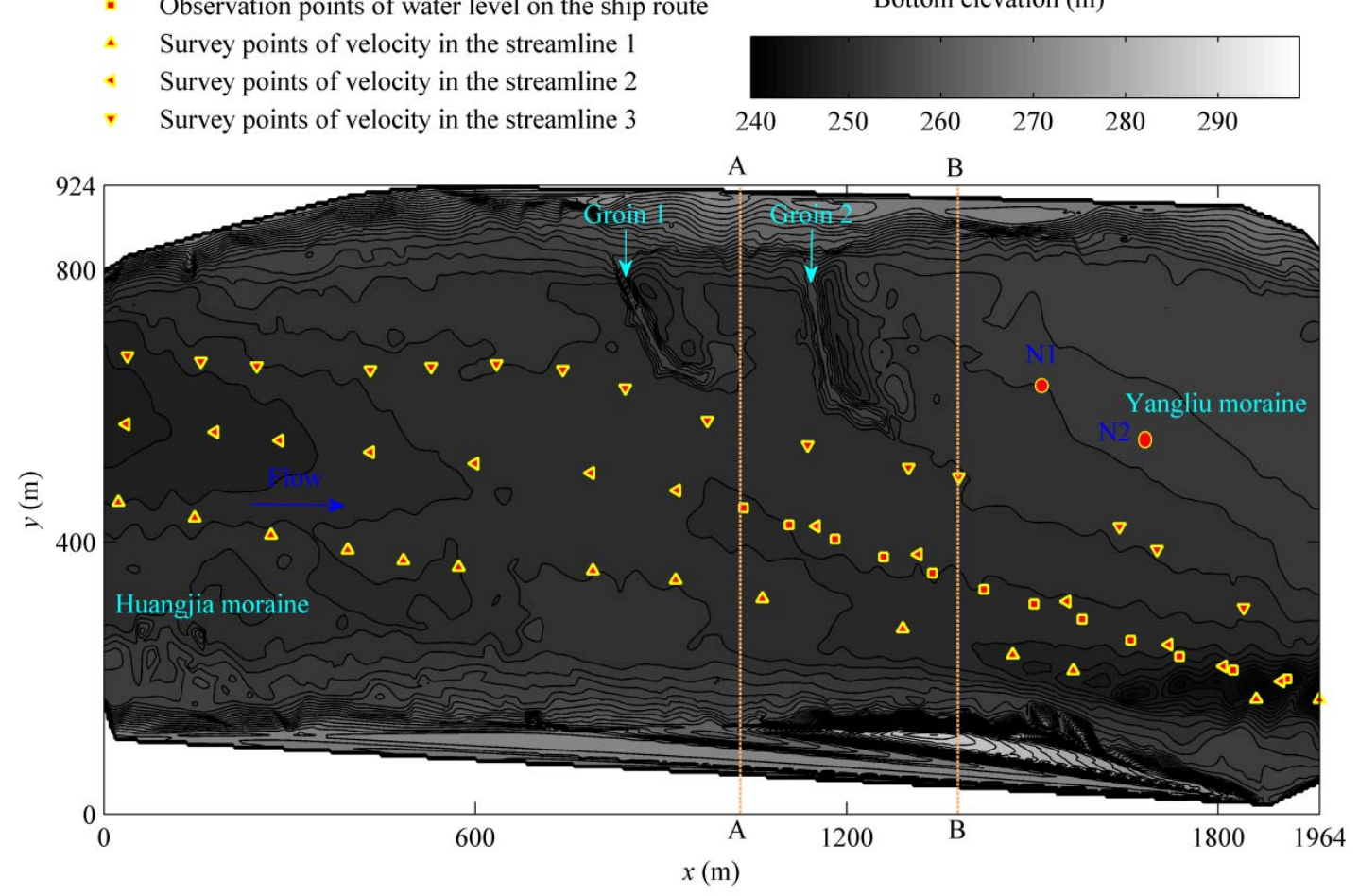

Bottom elevation (m) 


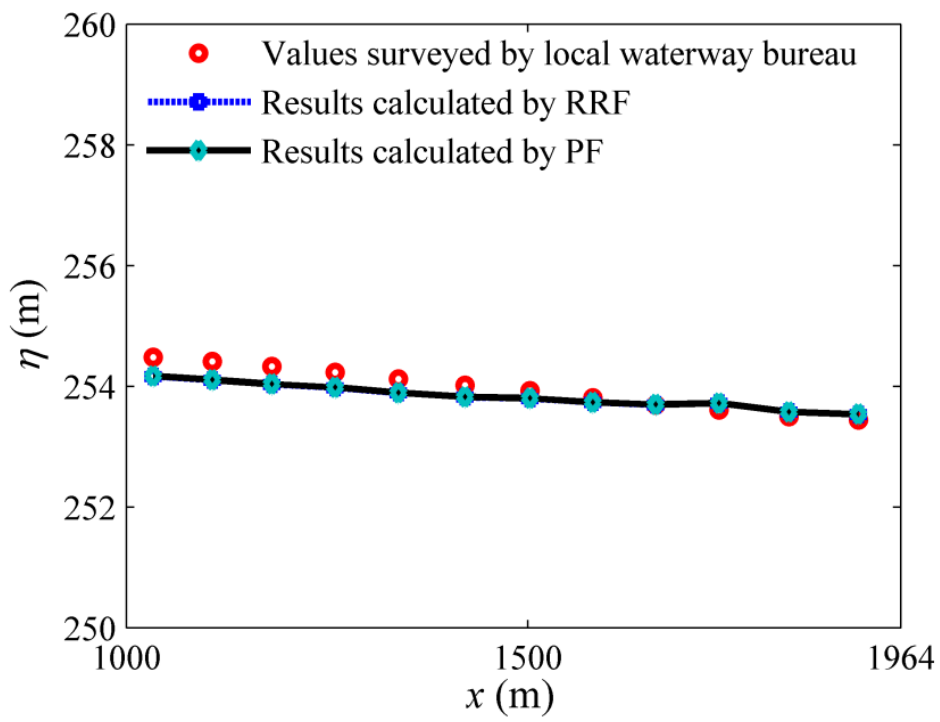

858

859 Figure 11 - Water level comparison between observed data and computed results on the ship 860 route.

861 

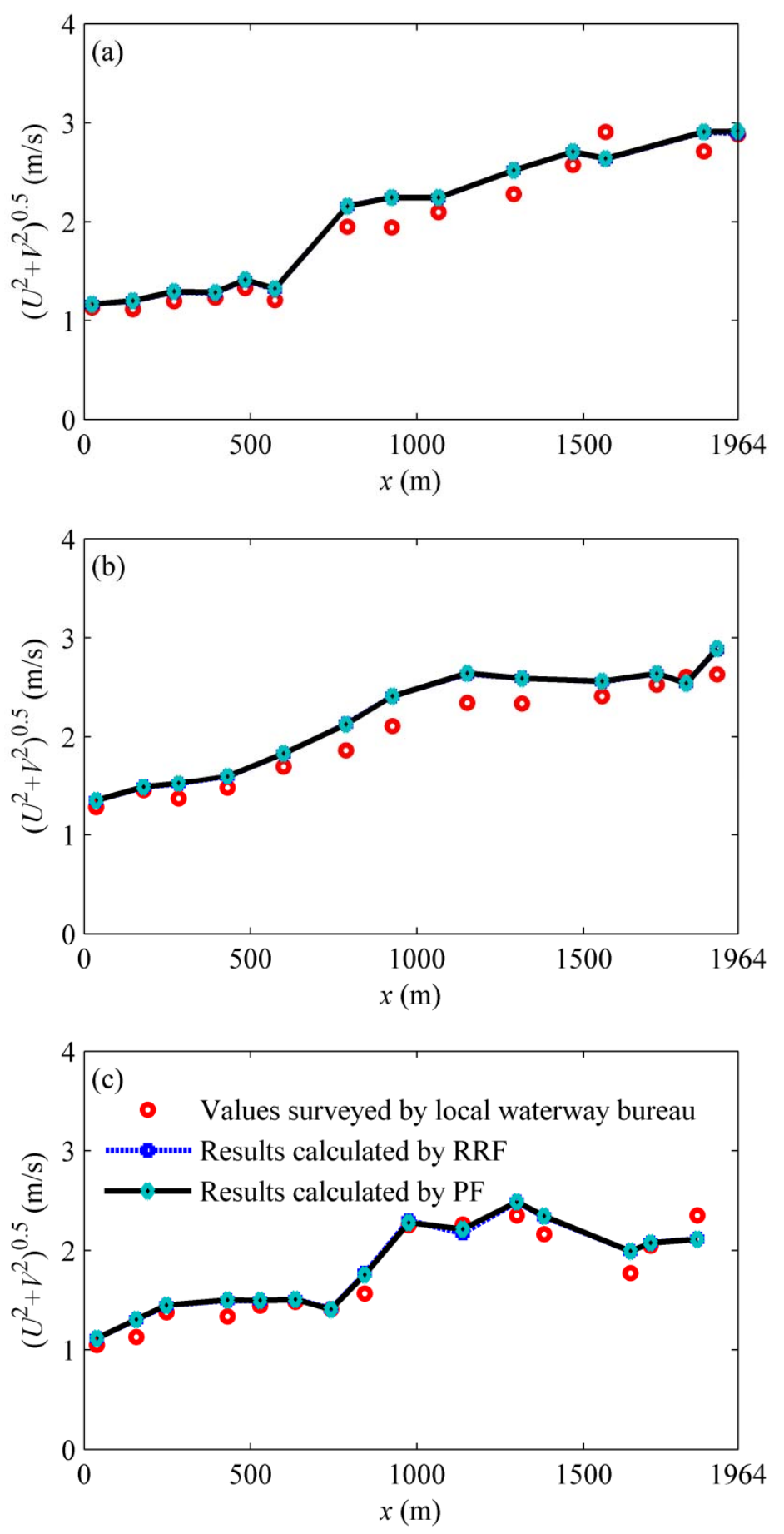

863

864 Figure 12 - Flow velocity comparison between measured values and calculated results in the 865 three streamlines: (a) streamline 1, (b) streamline 2 and (c) streamline 3. 


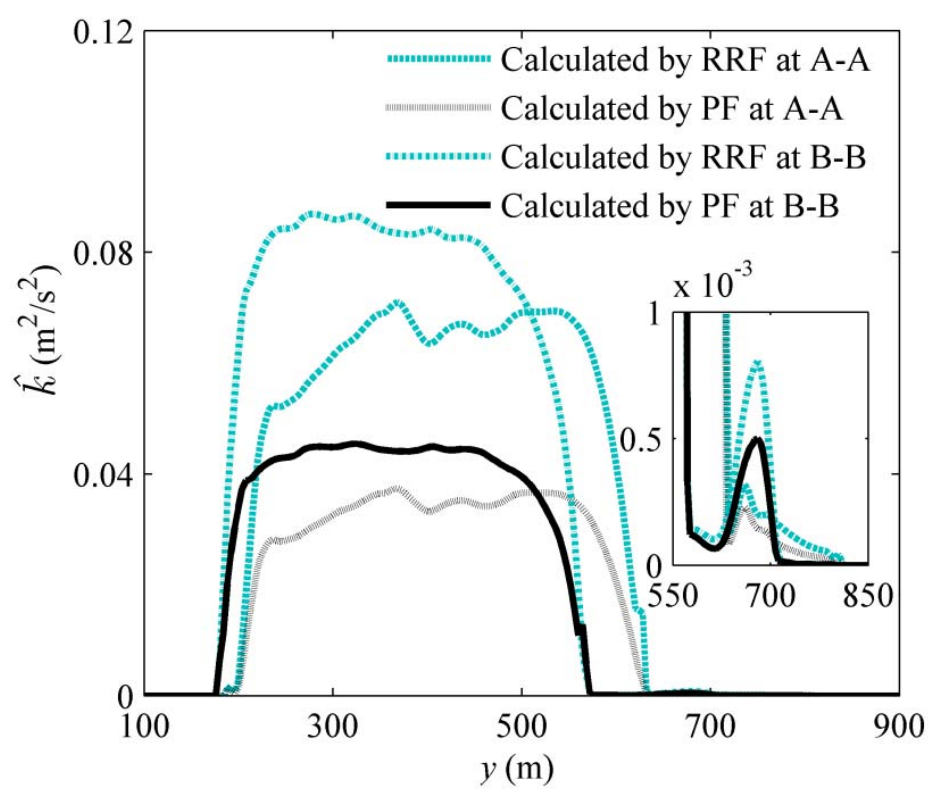

869 Figure 13 - Comparisons of the numerical results between PF and RRF at the sections of A-A 870 and B-B behind the two groins. 

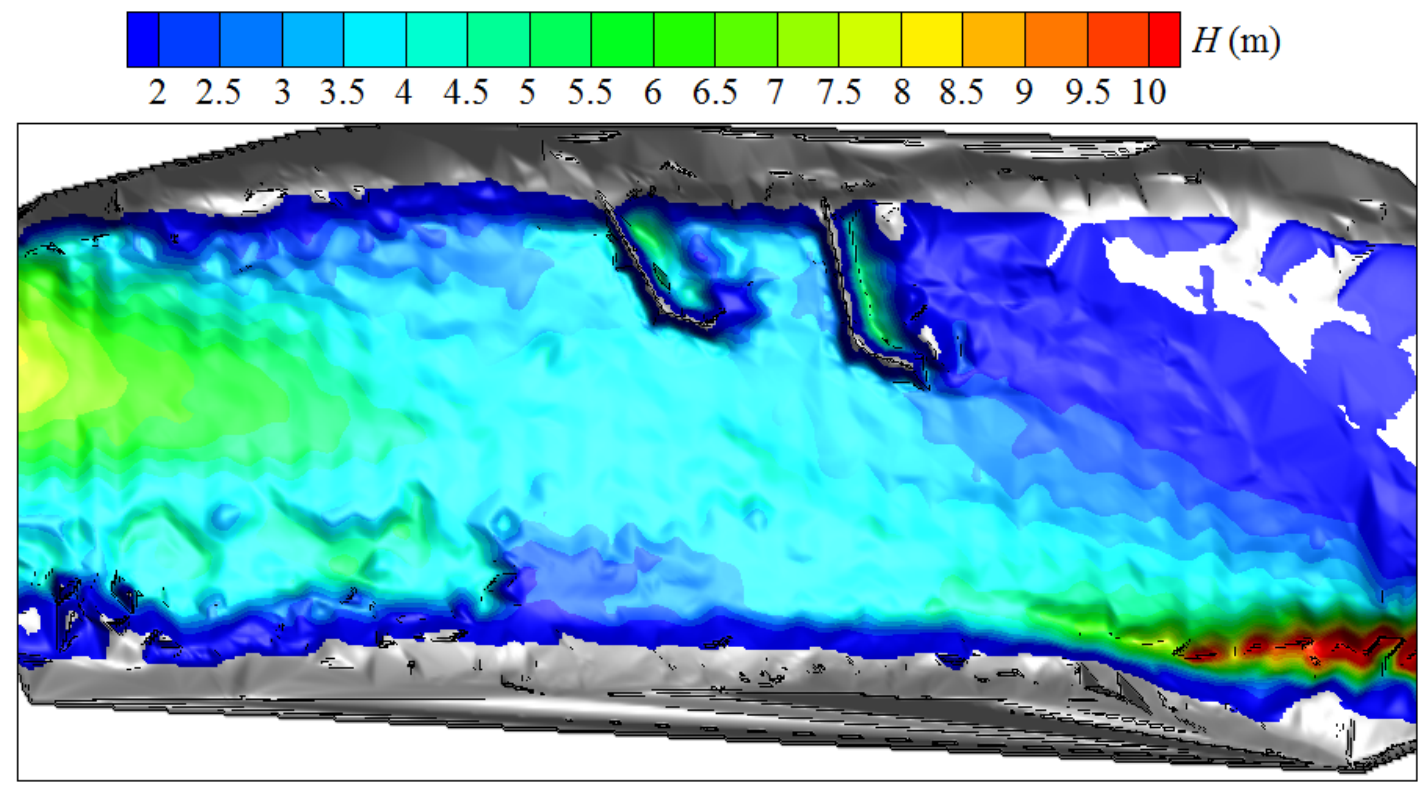

874 Figure 14 - Water depth calculated by the proposed model. 
876

877 Table 1. Hydraulic data collected with the experiments and numerically calculated.

\begin{tabular}{|c|c|c|c|c|c|c|c|c|c|c|}
\hline \multirow[t]{2}{*}{ Case } & \multirow{2}{*}{$\begin{array}{l}k_{\mathrm{s}} \\
\mathrm{mm}\end{array}$} & \multirow{2}{*}{$\begin{array}{l}H \\
\mathrm{~cm}\end{array}$} & \multirow{2}{*}{$\begin{array}{l}U_{0} \\
\mathrm{~cm} / \mathrm{s}\end{array}$} & \multirow[t]{2}{*}{$R e$} & \multirow[t]{2}{*}{$F r$} & \multirow{2}{*}{$\begin{array}{c}\hat{k} \text { acquired } \\
\text { from } \\
\text { measurements } \\
\left(\times 10^{-3}\right) \mathrm{m}^{2} / \mathrm{s}^{2}\end{array}$} & \multicolumn{2}{|c|}{$\hat{k}$ simulated by $\mathrm{PF}$} & \multicolumn{2}{|c|}{$\hat{k}$ simulated by RRF } \\
\hline & & & & & & & $\begin{array}{c}\text { Results } \\
\left(\times 10^{-3}\right) \mathrm{m}^{2} / \mathrm{s}^{2}\end{array}$ & $\begin{array}{l}\text { Relative } \\
\text { Absolute } \\
\text { Error \% }\end{array}$ & $\begin{array}{c}\text { Results } \\
\left(\times 10^{-3}\right) \mathrm{m}^{2} / \mathrm{s}^{2}\end{array}$ & $\begin{array}{c}\text { Relative } \\
\text { Absolute } \\
\text { Error \% }\end{array}$ \\
\hline $\mathrm{C} 1$ & 0.02 & 50.2 & 34.0 & 170,680 & 0.15 & 2.83 & 0.33 & 88.3 & 0.77 & 72.8 \\
\hline $\mathrm{C} 2$ & 2 & 42.0 & 36.2 & 152,040 & 0.18 & 2.36 & 0.70 & 70.3 & 1.40 & 40.7 \\
\hline $\mathrm{C} 3$ & 5 & 24.8 & 65.3 & 161,944 & 0.42 & 3.41 & 3.20 & 6.2 & 5.83 & 71.0 \\
\hline $\mathrm{C} 4$ & 10 & 19.6 & 46.3 & 90,748 & 0.33 & 1.85 & 2.04 & 10.3 & 3.51 & 89.7 \\
\hline $\mathrm{C} 5$ & 10 & 30.7 & 57.8 & 177,446 & 0.33 & 3.81 & 2.84 & 25.5 & 5.02 & 31.8 \\
\hline C6 & 10 & 19.4 & 101.0 & 195,940 & 0.73 & 5.27 & 8.48 & 60.9 & 14.69 & 178.7 \\
\hline C7 & 20 & 31.8 & 42.7 & 135,786 & 0.24 & 2.35 & 1.84 & 21.7 & 3.12 & 32.8 \\
\hline $\mathrm{C} 8$ & 20 & 28.1 & 63.5 & 178,435 & 0.38 & 2.45 & 4.14 & 69.0 & 6.96 & 184.1 \\
\hline C9 & 40 & 28.8 & 35.4 & 101,952 & 0.21 & 0.89 & 1.55 & 74.2 & 2.49 & 179.8 \\
\hline $\mathrm{C} 10$ & 40 & 29.5 & 62.5 & 184,375 & 0.37 & 3.24 & 4.70 & 45.1 & 7.60 & 134.6 \\
\hline C11 & 40 & 17.5 & 110.5 & 193,375 & 0.84 & 7.22 & 12.63 & 74.9 & 20.00 & 177.0 \\
\hline
\end{tabular}

$878 \quad k_{\mathrm{s}}$ : gravel median diameter $d_{50}, H$ and $U_{0}:$ average vertical distance from gravel bed to

879 water surface and bulk free-stream velocity in the middle section of the experimental flume,

880 respectively.

881 